\title{
$K$-THEORY OF LOCALLY COMPACT MODULES OVER RINGS OF INTEGERS
}

\author{
OLIVER BRAUNLING
}

\begin{abstract}
We generalize a recent result of Clausen: For a number field with integers $\mathcal{O}$, we compute the $K$-theory of locally compact $\mathcal{O}$-modules. For the rational integers this recovers Clausen's result as a special case. Our method of proof is quite different: Instead of a homotopy coherent cone construction in $\infty$-categories, we rely on calculus of fraction type results in the style of Schlichting. This produces concrete exact category models for certain quotients, a fact which might be of independent interest. As in Clausen's work, our computation works for all localizing invariants, not just $K$-theory.
\end{abstract}

\section{INTRODUCTION}

Let LCA denote the category of locally compact abelian (LCA) groups, Cat ${ }_{\infty}^{\text {ex }}$ the $\infty$-category of small stable $\infty$-categories, A any stable $\infty$-category. Recently, Dustin Clausen proved the following theorem:

Theorem (Clausen). For every localizing invariant $K: \mathrm{Cat}_{\infty}^{\mathrm{ex}} \rightarrow \mathrm{A}$, there is a canonical fiber sequence

$$
K(\mathbb{Z}) \longrightarrow K(\mathbb{R}) \longrightarrow K(\mathrm{LCA})
$$

where the first map is induced from the ring homomorphism $\mathbb{Z} \rightarrow \mathbb{R}$.

This is [Cla17, Theorem 3.4]. Clausen's work is based on earlier ideas of Hoffmann and Spitzweck HS07. As an example, we can take non-connective $K$-theory for $K$ (the letter was chosen suggestively), which has values in spectra, and obtain a computation of the $K$-theory of the category LCA.

We generalize Clausen's result as follows:

Theorem 1.1. Let $F$ be a number field and $\mathcal{O}$ its ring of integers. Let $\mathrm{LCA}_{\mathcal{O}}$ be the category of locally compact $\mathcal{O}$-modules. For every localizing invariant $K: \mathrm{Cat}_{\infty}^{\mathrm{ex}} \rightarrow \mathrm{A}$, there is a canonical fiber sequence

$$
K(\mathcal{O}) \longrightarrow K(\mathbb{R})^{r} \oplus K(\mathbb{C})^{s} \longrightarrow K\left(\mathrm{LCA}_{\mathcal{O}}\right)
$$

where $r$ is the number of real places and $s$ the number of complex places.

See Theorem 4.5 below for details. For $\mathcal{O}=\mathbb{Z}$, the category LCA $\mathcal{O}$ agrees with the category LCA, so we get the same statement as in Clausen's result. Beyond this, the category LCA $\mathcal{O}_{0}$ appears to reflect some phenomena around the infinite primes in a very natural way. This is quite remarkable since many such theories require some manual handling for infinite primes.

\footnotetext{
2000 Mathematics Subject Classification. Primary 22B05; Secondary 19D10.

Key words and phrases. Locally compact abelian groups, LCA groups, Lichtenbaum conjectures, infinite primes.

The author was supported by DFG GK1821 "Cohomological Methods in Geometry" and a Junior Fellowship at the Freiburg Institute for Advanced Studies (FRIAS).
} 
The category $L C A_{\mathcal{O}}$ is of glaring beauty: $L C A_{\mathcal{O}}$ is a quasi-abelian exact category with duality. The Minkowski embedding gives rise to an exact sequence

$$
\mathcal{O} \hookrightarrow \bigoplus_{\sigma \in S} \mathbb{R}_{\sigma} \rightarrow \mathbb{T}_{\mathcal{O}}
$$

( $S$ the set of infinite places, $\mathbb{R}_{\sigma}$ the codomain of $\sigma$, and $\mathbb{T}_{\mathcal{O}}$ the torus quotient). In LCA $_{\mathcal{O}}$ this sequence is simultaneously, (1) an injective resolution of $\mathcal{O},(2)$ a projective resolution of $\mathbb{T}_{\mathcal{O}}$, and (3) the Pontryagin dual of the sequence is isomorphic to the sequence itself. So the Minkowski embedding is hardcoded in the homological algebra of LCA ${ }_{\mathcal{O}}$.

Similarly for the Dirichlet embedding: For algebraic $K$-theory, the sequence in Theorem 1.1 in degree one yields

$$
K_{1}(\mathcal{O}) \longrightarrow\left(\mathbb{R}^{\times}\right)^{r} \oplus\left(\mathbb{C}^{\times}\right)^{s} \longrightarrow K_{1}\left(\mathrm{LCA}_{\mathcal{O}}\right) \longrightarrow \mathrm{Cl}(\mathcal{O}) \longrightarrow 0
$$

which essentially identifies $K_{1}\left(\mathrm{LCA}_{\mathcal{O}}\right)$ as an extension of the class group with $\mathbb{R}_{>0}^{\times} \times($torus), naturally containing the torus arising from Dirichlet's Unit Theorem. The free real factor $\mathbb{R}_{>0}^{\times}$ corresponds to choosing a normalization for the Haar measure the underlying LCA group.

These facts resemble regulator constructions. Indeed, making use of properties of the Borel regulator, we get:

Theorem 1.2. Let $F$ be a number field, $\mathcal{O}$ its ring of integers, and $K$ (non-connective) algebraic $K$-theory. With rational coefficients, the long exact sequence induced from the theorem above splits into short exact sequences

$$
0 \rightarrow K_{n}(\mathcal{O})_{\mathbb{Q}} \rightarrow K_{n}(\mathbb{R})_{\mathbb{Q}}^{r} \oplus K_{n}(\mathbb{C})_{\mathbb{Q}}^{s} \rightarrow K_{n}\left(\mathrm{LCA}_{\mathcal{O}}\right)_{\mathbb{Q}} \rightarrow 0
$$

for all $n$.

See Theorem 5.1. Our methods are quite different from Clausen's in [Cla17. While Clausen sets up a homotopy coherent cone construction in the context of stable $\infty$-categories, this paper exclusively deals with exact categories. Our method is based on techniques of Schlichting (namely left and right $s$-filtering subcategories) in order to show that certain quotient constructions do not only make sense on a derived level, but admit concrete exact category models. The main technical tool is the following:

Theorem 1.3. Let $F$ be a number field, $\mathcal{O}$ its ring of integers. Then

(1) The compactly generated $\mathcal{O}$-modules $\mathrm{LCA}_{\mathcal{O}, c g}$ are left s-filtering in $\mathrm{LCA}_{\mathcal{O}}$.

(2) The $\mathcal{O}$-modules $\mathrm{LCA}_{\mathcal{O}, \text { nss }}$ without small subgroups are right s-filtering in $\mathrm{LCA}_{\mathcal{O}}$.

These results essentially amount to setting up a calculus of left or right fractions with certain additional properties. They might be of independent interest. Thus, even in the case $\mathcal{O}=\mathbb{Z}$, our proof is different from the one in [Cla17.

Needless to say, this article is heavily inspired by Clausen's work.

Conventions: We shall work a lot with exact categories. We follow the nomenclature of

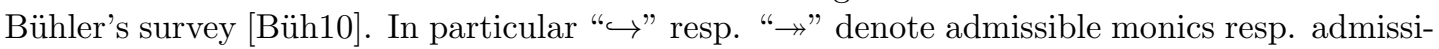
ble epics with respect to the exact structure. For stable $\infty$-categories and localizing invariants, we employ the conventions of [BGT13.

\section{The CATEgory of LOCALly COMPACT MODUleS}

Let LCA be the category of locally compact abelian groups with continuous group homomorphisms as its morphisms. Hoffmann and Spitzweck have observed that LCA is a quasi-abelian category [HS07], and thus an exact category. This can be generalized as follows: 
Definition 2.1. Let $R$ be a commutative unital ring. Regard $R$ as equipped with the discrete topology. Let $\mathrm{LCA}_{R}$ be the category of locally compact $R$-modules with continuous $R$-module homomorphisms as morphisms.

This means that an object in $\mathrm{LCA}_{R}$ is (1) an $R$-module $M,(2)$ that its additive group $(M,+)$ comes equipped with the structure of an LCA group, and (3) the constraint that we demand each element of $R$ to act on $(M,+)$ via a continuous endomorphism.

Example 1. We have $L_{C A}=L C A$, i.e. this definition encompasses the category of LCA groups as a special case. The full subcategory of discrete $R$-modules in LCA $_{R}$ is literally isomorphic to the category of all $R$-modules.

Proposition 2.2. The category $\mathrm{LCA}_{R}$ is quasi-abelian.

Proof. The proof of Hoffmann and Spitzweck for $R=\mathbb{Z}$ carries over verbatim, HS07, Prop. $1.2]$.

In particular, LCA $_{R}$ is an exact category in a natural way [Büh10, Prop. 4.4]. With respect to this structure, the admissible monics are the closed injective $R$-module homomorphisms, while the admissible epics are open surjective $R$-module homomorphisms. All kernels and cokernels exist; in particular the category is idempotent complete so that arguments like Büh10, Cor. 7.7] apply. The category is never abelian.

Recall that every morphism $f: A \rightarrow B$ in a quasi-abelian category $C$ has a canonical factorization

$$
A \rightarrow \operatorname{coim}_{\mathrm{C}}(f) \stackrel{r}{\rightarrow} \operatorname{im}_{\mathrm{C}}(f) \hookrightarrow B,
$$

where $\operatorname{coim}_{C}(f):=A / \operatorname{ker}(f)$ is called the coimage. In an abelian category one has the luxury of $r$ being an isomorphism. This is usually false for quasi-abelian categories and false for LCA $R$ in particular.

Example 2. The inclusion $\mathbb{Q} \rightarrow \mathbb{R}$ is a monic in LCA, but not an admissible monic. The coimage is $\mathbb{Q}$, while the image is $\mathbb{R}$. The Pontryagin dual is $\mathbb{R} \rightarrow \mathbb{A}_{\mathbb{Q}} / \mathbb{Q}$, where $\mathbb{A}_{\mathbb{Q}}$ denotes the rational adèles. This map is an epic, but not an admissible epic. The quotient $\mathbb{A}_{\mathbb{Q}} / \mathbb{Q}$ is a compact connected $\mathbb{Q}$-vector space.

Example 3 . If $\mathbb{R}_{\delta}$ denotes the reals with the discrete topology, $\mathbb{R}_{\delta} \rightarrow \mathbb{R}$ is a morphism in LCA. It is both monic and epic, yet not an isomorphism. It is neither an admissible monic nor an admissible epic. Its Pontryagin dual is $\mathbb{R} \rightarrow b \mathbb{R}$, the map underlying the Bohr compactification.

Notation. In the literature for topological groups the notation $\operatorname{im}(f)$ usually refers to the settheoretic image. In the present article we run into the trouble that this is not the same as the image object $\operatorname{im}_{\mathrm{LCA}}(f)$ in the category LCA. However, it feels more than awkward to write $\operatorname{coim}_{\mathrm{LCA}}(f)$, which would be the appropriate term from the viewpoint of category theory. For this reason, we shall always write $\operatorname{im}_{\text {Set }}(f)$ and speak of the set-theoretic image, as a compromise to create a both category-theoretically correct yet reader-friendly text.

We return to LCA. We denote the circle group by $\mathbb{T}:=\mathbb{R} / \mathbb{Z}$ and write $G^{\vee}:=\operatorname{Hom}_{\text {cts }}(G, \mathbb{T})$ for the Pontryagin dual, where the homomorphism group is equipped with the compact-open topology. Pontryagin duality then yields an exact anti-equivalence

$$
(-)^{\vee}: \text { LCA }^{o p} \stackrel{\sim}{\longrightarrow} \text { LCA. }
$$

If $f: G \rightarrow H$ is injective, then $f^{\vee}: H^{\vee} \rightarrow G^{\vee}$ has dense set-theoretic image; if $f$ has dense set-theoretic image, $f^{\vee}$ is injective. If $f$ is an admissible monic, $f^{\vee}$ is an admissible epic, and conversely. 
This generalizes to $\mathrm{LCA}_{R}$ as follows: Although $\mathbb{T}$ does not carry a natural $R$-module structure in any way and thus only makes sense in LCA, the dual $G^{\vee}$ of an $R$-module again carries an $R$-module structure by

$$
(a \cdot \chi)(m):=\chi(a \cdot m) \quad \text { for } \quad a \in R, \chi \in G^{\vee}, m \in G .
$$

This leads to a generalized form of Pontryagin duality:

Theorem 2.3. There is an exact anti-equivalence of exact categories

$$
(-)^{\vee}: \mathrm{LCA}_{R}^{o p} \stackrel{\sim}{\longrightarrow} \mathrm{LCA}_{R}
$$

reflexive in the sense that the natural morphism $\eta_{G}: G \stackrel{\sim}{\rightarrow} G^{\vee \vee}$ is an isomorphism. On the level of the underlying additive groups, this agrees with ordinary Pontryagin duality. In particular, it sends compact $R$-modules to discrete $R$-modules, and conversely.

This extension of Pontryagin duality has been observed by a number of people in various versions, e.g., Stöhr [Sto69] and Levin Lev73, Theorem 1].

In fact, we can combine the exact structure with this duality:

Proposition 2.4. The category $\mathrm{LCA}_{R}$, equipped with Pontryagin duality, is an exact category with duality (in the sense of [Sch10, Definition 2.1]).

Of course this is precisely what everyone would expect, yet I know of no literature studying the category LCA from the perspective of an exact category with duality.

Proof. Standard.

We return to LCA:

Definition 2.5. Let $G$ be an $L C A$ group. A subset $U \subseteq G$ is called symmetric if it is closed under taking inverses.

(1) The group $G$ has no small subgroups if there exists a symmetric open neighbourhood $U \subset G$ of the neutral element such that $U$ contains no non-trivial subgroup.

(2) The group $G$ is compactly generated if there exists a compact symmetric neighbourhood $U \subset G$ of the neutral element such that $G=\bigcup_{n \geq 1} U^{n}$, where $U^{n}$ denotes the image of the product of any $n$ elements in $U$.

Let $\mathrm{LCA}_{n s s}$ (resp. $\mathrm{LCA}_{c g}$ ) be the full subcategory of groups having no small subgroups (resp. being compactly generated).

The same qualifications make sense for objects in $\mathrm{LCA}_{R}$. We write $\mathrm{LCA}_{R, n s s}$ resp. LCA $R, c g$ for those objects whose underlying additive groups have no small subgroups resp. are compactly generated.

Just as compact and discrete groups are exchanged under Pontryagin duality, so are the above concepts:

Proposition 2.6. The category $\mathrm{LCA}_{R, n s s}$ (resp. $\mathrm{LCA}_{R, c g}$ ) is extension-closed in $\mathrm{LCA}_{R}$. Moreover, both are dual partners under Pontryagin duality, i.e. $\mathrm{LCA}_{R}^{o p} \longrightarrow \mathrm{LCA}_{R}$ sends $\mathrm{LCA}_{R, n s s}^{\text {op }}$ to $\mathrm{LCA}_{R, c g}$ and conversely.

Proof. This is proven in Moskowitz for $R=\mathbb{Z}$, see [Mos67, Theorem 2.6]. However, since these qualities only rely on the topology of the underlying additive group, this is sufficient in our situation as well. 
2.1. Number fields. Let $F$ be a number field and $\mathcal{O}$ its ring of integers. Regard $\mathcal{O}$ as equipped with the discrete topology. Then $\mathrm{LCA}_{\mathcal{O}}$ is the category of locally compact $\mathcal{O}$-modules with continuous $\mathcal{O}$-module homomorphisms as morphisms.

Suppose

$$
\sigma: F \longrightarrow \mathbb{R} \text { (resp. } \mathbb{C})
$$

is a real or complex embedding of the number field (i.e. $\sigma$ is a ring homomorphism).

Definition 2.7. We define $\mathbb{R}_{\sigma}$ to be the $\mathcal{O}$-module whose underlying additive group is $\mathbb{R}$ (resp. $\mathbb{C})$ and whose $\mathcal{O}$-module structure is given by

$$
a \cdot \beta:=\sigma(a) \beta \quad \text { for } \quad a \in \mathcal{O}, \beta \in \mathbb{R} \text { (resp. } \beta \in \mathbb{C}) .
$$

Note that we write $\mathbb{R}_{\sigma}$ even if $\sigma$ comes from a strictly complex embedding. This is convenient in order to avoid repeatedly having to go through case distinctions for real and complex places. It is easy to see that $\mathbb{R}_{\sigma} \in \mathrm{LCA} \mathrm{O}_{\mathcal{O}}$. We have isomorphy

$$
\mathbb{R}_{\sigma}^{\vee} \simeq \mathbb{R}_{\sigma}
$$

even though there is no canonical isomorphism.

We recall that an LCA group is called a vector group if it admits an isomorphism to $\mathbb{R}^{n}$ for some $n \geq 0$. We shall call $G$ in $\mathrm{LCA}_{\mathcal{O}}$ a vector $\mathcal{O}$-module if its underlying additive group is a vector group. The following result goes back to Levin [Lev73].

Proposition 2.8. If $G \in \mathrm{LCA}_{\mathcal{O}}$ is a vector $\mathcal{O}$-module, $G$ is a finite direct sum $G \simeq \bigoplus_{\sigma \in I} \mathbb{R}_{\sigma}$, where $I$ is a finite list of real and complex places.

We write "list" with the meaning that repetitions are allowed.

Proof. (follows Levin, Part 4 of the proof of Lev73, Prop. 1]) We give a quick sketch, as this argument plays a crucial rôle: By a standard argument, every LCA endomorphism of a vector group is $\mathbb{R}$-linear (Sketch: it is an abelian group homomorphism; then since $G$ is uniquely divisible, it must be $\mathbb{Q}$-linear and by continuity must be $\mathbb{R}$-linear). Thus, $\mathcal{O}$ acts by $\mathbb{R}$-vector space endomorphisms on $G$. Let $\lambda \in F$ be a primitive element of the number field so that $F=\mathbb{Q}(\lambda)$. Without loss of generality, we may demand $\lambda \in \mathcal{O}$; let $f$ be its minimal polynomial. Then the $\mathcal{O}$-module structure is entirely determined by $\mathbb{R}$-linearity and the action of $\lambda$, so $G$ canonically is an $\mathbb{R}[T] /(f)$-module, where $T$ acts as $T(m):=\lambda \cdot m$. So the $\mathcal{O}$-module structure uniquely defines an $\mathbb{R}[T] /(f)$-module structure. Conversely, every $\mathbb{R}[T] /(f)$-module is an $F$ vector space and thus an $\mathcal{O}$-module. We see that a direct sum decomposition of $G$ as an $\mathbb{R}[T] /(f)$-module agrees with a direct sum decomposition as $\mathcal{O}$-modules; and summands are simple in one sense iff they are simple in the other. Polynomials over $\mathbb{R}$ factor in linear and quadratic factors, and these correspond precisely to the real and complex embeddings; e.g. in the first case the simple summands have the shape $\mathbb{R}[T] /(T-\sigma(\lambda))$; but this is just $\mathbb{R}_{\sigma}$; and correspondingly for complex embeddings.

Corollary 2.9. Let $\mathrm{LCA}, \mathbb{R}$ be the full subcategory of $\mathrm{LCA}_{\mathcal{O}}$ whose objects are vector $\mathcal{O}$-modules. This is an extension-closed subcategory. For every localizing invariant $K: \mathrm{Cat}_{\infty}^{\mathrm{ex}} \rightarrow \mathrm{A}$, there are canonical equivalences

$$
K\left(\mathrm{LCA}_{\mathcal{O}, \mathbb{R}}\right) \cong K(\mathbb{R})^{r} \oplus K(\mathbb{C})^{s},
$$

where $r$ is the number of real places of $F$, and $s$ the number of complex places.

Proof. As in the previous proof, we may equivalently classify finitely generated $\mathbb{R}[T] /(f)$ modules, where $f$ is a minimal polynomial of a primitive element of $F$ over the rationals. Thus, we have exact equivalences of exact categories

$$
\operatorname{LCA}_{\mathcal{O}, \mathbb{R}} \stackrel{\sim}{\longrightarrow} \operatorname{Mod}_{f g}(\mathbb{R}[T] /(f)) \stackrel{\sim}{\longrightarrow} \prod_{i} \operatorname{Mod}_{f g}\left(\mathbb{R}[T] /\left(f_{i}\right)\right),
$$


where $f_{i}$ are the irreducible factors so that $\mathbb{R}[T] /(f) \cong \prod_{i} \mathbb{R}[T] /\left(f_{i}\right) \cong \mathbb{R}^{r} \times \mathbb{C}^{s}$ holds as an isomorphism of rings.

Definition 2.10. We introduce some further categories:

(1) Let $\mathrm{LCA}_{\mathcal{O}, \mathbb{R} C}$ be the full subcategory of $\mathrm{LCA}_{\mathcal{O}}$ of objects which admit an isomorphism to $V \oplus C$ for some vector $\mathcal{O}$-module $V$ and compact $\mathcal{O}$-module $C$.

(2) Let $\mathrm{LCA}_{\mathcal{O}, \mathbb{R} D}$ be the full subcategory of $\mathrm{LCA}_{\mathcal{O}}$ of objects which admit an isomorphism to $V \oplus D$ for some vector $\mathcal{O}$-module $V$ and discrete $\mathcal{O}$-module $D$.

We have the full subcategory inclusions

$$
\mathrm{LCA}_{\mathcal{O}, \mathbb{R} C} \subset \mathrm{LCA}_{\mathcal{O}, c g} \quad \text { and } \quad \mathrm{LCA}_{\mathcal{O}, \mathbb{R} D} \subset \mathrm{LCA}_{\mathcal{O}, n s s} .
$$

Lemma 2.11 ([Lev73, Lemma 2]). Suppose the underlying additive group of an object $G \in$ $\mathrm{LCA}_{\mathcal{O}}$ admits a direct sum splitting

$$
G \simeq H \oplus D \quad \text { in } \quad \text { LCA }
$$

with $H \simeq \mathbb{R}^{n} \oplus \mathbb{T}^{m}$ for some $n, m \geq 0$ finite, and $D$ discrete. Then

(1) $H$ and $D$ are closed under $\mathcal{O}$-multiplication, so that $H, D \in \mathrm{LCA}_{\mathcal{O}}$ canonically, and

(2) the given splitting comes from a direct sum splitting in $\mathrm{LCA}_{\mathcal{O}}$ and $H$ is a vector $\mathcal{O}$ module.

Proof. Note that $H$ is the connected component of zero in $G$. As multiplication by an element $a \in \mathcal{O}$ acts continuously, the image of a connected set is connected, so it must lie in $H$ again. Thus, the subgroup $H$ is closed under the action of $\mathcal{O}$ and thus $H \subseteq G$ is a topological $\mathcal{O}$ submodule. Next, $H$ is divisible as a $\mathbb{Z}$-module, and thus divisible as an algebraic $\mathcal{O}$-module. Since $\mathcal{O}$ is a Dedekind domain, this implies that $H$ is an injective algebraic $\mathcal{O}$-module, i.e. it is a direct summand of $G$ on the level of the underlying $\mathcal{O}$-module. The complement $D$ thus is also closed under the action of $\mathcal{O}$. Since $D$ is discrete, this action is tautologically also continuous. Thus, $H, D \in \mathrm{LCA}_{\mathcal{O}}$ and the direct sum decomposition holds in $\mathrm{LCA}_{\mathcal{O}}$.

Every ideal $J$ of $\mathcal{O}$ can be equipped with the discrete topology and thus defines an object of $\mathrm{LCA}_{\mathcal{O}}$ in a natural way. We shall tacitly write $J \in \mathrm{LCA}_{\mathcal{O}}$. Recall that under the Minkowski embedding

$$
M: \mathcal{O} \longrightarrow \bigoplus_{\sigma \in S} \mathbb{R}_{\sigma}
$$

where $\sigma$ runs through all real and complex places, a non-trivial ideal (or more generally: fractional ideal) $J$ is sent to a full rank lattice in a vector group. Note that $M$ is also an $\mathcal{O}$-module homomorphism; continuous since $\mathcal{O}$ is discrete. Thus, we get an exact sequence in LCA $\mathrm{O}_{\text {, }}$

$$
J \hookrightarrow \bigoplus_{\sigma \in S} \mathbb{R}_{\sigma} \rightarrow \mathbb{T}_{J}
$$

where $\mathbb{T}_{J}$ is our notation for the quotient. We call it the Minkowski sequence of $J$. Of course, the underlying LCA group of $\mathbb{T}_{J}$ is a torus, justifying the notation. Note further that under Pontryagin duality this sequence is self-dual, in that

$$
J^{\vee} \leftarrow \bigoplus_{\sigma \in S} \mathbb{R}_{\sigma}^{\vee} \hookleftarrow \mathbb{T}_{J}^{\vee}
$$

is non-canonically isomorphic to the previous exact sequence.

Next, we classify the admissible subobjects of vector $\mathcal{O}$-modules.

Proposition 2.12. Suppose $G \in \mathrm{LCA}_{\mathcal{O}}$ is a vector $\mathcal{O}$-module and $\alpha: H \hookrightarrow G$ an admissible monic. Then $\alpha$ can be written as a finite $\mathcal{O}$-linear direct sum of morphisms 
(1) $0 \hookrightarrow \mathbb{R}_{\sigma}$, for $\sigma$ a real or complex place,

(2) $\mathbb{R}_{\sigma} \stackrel{1}{\rightarrow} \mathbb{R}_{\sigma}$ for $\sigma$ a real or complex place,

(3) $J \hookrightarrow \bigoplus_{\sigma} \mathbb{R}_{\sigma}$ the Minkowski embedding of Equation [2.1.

Proof. We work on the level of the underlying additive LCA group at first: Then by our assumptions $G \simeq \mathbb{R}^{n}$ for some $n \geq 0$ in LCA and $H$ is a closed subgroup. By the classification of such closed subgroups, Mor77, Theorem 6], there exists an $\mathbb{R}$-vector space basis $x_{1}, \ldots, x_{n}$ of $\mathbb{R}^{n}$ and some integers $0 \leq p \leq q \leq n$ such that

$$
H=\mathbb{Z}\left\langle x_{1}, \ldots, x_{p}\right\rangle \oplus \mathbb{R}\left\langle x_{p+1}, \ldots, x_{q}\right\rangle \subseteq G,
$$

where the $\mathbb{Z}$-part sits in $\mathbb{R}\left\langle x_{1}, \ldots, x_{p}\right\rangle$ as a discrete full rank lattice. So far, this is only a direct sum in LCA, however by Lemma 2.11 it must come from a direct sum decomposition in LCA ${ }_{\mathcal{O}}$. Hence, the second summand must be a vector $\mathcal{O}$-module, and thus is described by Proposition 2.8. and the former a discrete $\mathcal{O}$-module, necessarily finitely generated as it is module-finite over $\mathbb{Z}$. Hence, in $\mathrm{LCA}_{\mathcal{O}}$ the admissible monic $\alpha: H \hookrightarrow G$ has the shape

$$
\alpha: M \oplus \coprod_{\sigma \in I_{1}} \mathbb{R}_{\sigma} \hookrightarrow \coprod_{\sigma \in I_{2}} \mathbb{R}_{\sigma}
$$

where $I_{1}, I_{2}$ are finite lists of real and complex places, and $M$ is a finitely generated $\mathcal{O}$-module, discretely embedded into the right-hand side as a lattice. The map originating from the summand $M$ factors over $M \rightarrow M \otimes_{\mathcal{O}} F$ since the vector $\mathcal{O}$-modules on the right are $F$-vector spaces. Hence, if $f \in \mathbb{Q}[T]$ denotes the minimal polynomial of a primitive element $\lambda \in F$, then this $\mathcal{O}$-module homomorphism is of the shape

$$
M \rightarrow M \otimes_{\mathcal{O}} \mathbb{Q}[T] /(f) \stackrel{\tilde{\alpha}}{\rightarrow} \coprod_{\sigma \in I_{2}} \mathbb{R}[T] /\left(f_{\sigma}\right),
$$

where we write $f_{\sigma}$ for the irreducible factor of $f$ over $\mathbb{R}$ corresponding to the place $\sigma$ (so quadratic resp. linear for real resp. complex places $\sigma \in I_{2}$ ); and $\tilde{\alpha}$ is an $F$-vector space homomorphism. As $\mathcal{O}$ is a Dedekind domain, and since $M$ is finitely generated torsion-free, $M$ must be projective and thus $M$ is isomorphic to a finite direct sum of ideals $J$ of $\mathcal{O}$. Thus, in order to prove our claim, it suffices to assume $M=J$ for $J$ such an ideal. Now proceed as follows: If the list $I_{2}$ in the morphism

$$
J \longrightarrow \coprod_{\sigma \in I_{2}} \mathbb{R}_{\sigma}
$$

has duplicates, then the image factors over the subobject given by $I_{2}$, but with all duplicates removed (this arises as a subobject through the diagonal map of the single copy, embedding diagonally into the summands of all replica of this single copy appearing in $I_{2}$ ). On the other hand, each real and complex place must appear in $I_{2}$ at least once: The discussion around Equation 2.1 implies that $J \rightarrow J \otimes_{\mathcal{O}} F$ is the embedding as a fractional ideal and since this is a full rank embedding, the standard results imply that any projection to a proper subset of embeddings cannot have discrete image. This means that its image in $G$ can only be discrete if all real and complex places $\sigma$ appear on the right at least once, letting $J \rightarrow G$ factor over a Minkowski embedding as in Equation 2.1. Thus, the map $\alpha$ is a direct sum of maps of the shape $\mathbb{R}_{\sigma} \stackrel{1}{\rightarrow} \mathbb{R}_{\sigma}$ and Minkowski embeddings $J \hookrightarrow \bigoplus_{\sigma \in I^{\prime}} \mathbb{R}_{\sigma}$ as in Equation 2.1 (where $I^{\prime}$ features each real and complex place exactly once).

Next, we shall use the following structure result due to Levin:

Proposition 2.13 ([Lev73, Prop. 1]). We have the following structure results for objects in $\mathrm{LCA}_{\mathcal{O}}$ : 
(1) Every object in $\mathrm{LCA}_{\mathcal{O}, \text { nss }}$ is isomorphic to $\bigoplus_{\sigma \in I} \mathbb{R}_{\sigma} \oplus \bigoplus_{J \in \mathcal{I}} \mathbb{T}_{J} \oplus D$ with $D$ discrete $\mathcal{O}$-module, $\mathcal{I}$ a finite list of ideals in $\mathcal{O}$, and $I$ a finite list of real and complex places.

(2) Every object in $\mathrm{LCA}_{\mathcal{O}, \text { cg }}$ is isomorphic to $\bigoplus_{\sigma \in I} \mathbb{R}_{\sigma} \oplus \bigoplus_{J \in \mathcal{I}} J \oplus C$ with $C$ a compact $\mathcal{O}$-module, $\mathcal{I}$ a finite list of ideals in $\mathcal{O}$, and $I$ a finite list of real and complex places.

Here an ideal $J$ is understood to carry the discrete topology, and $\mathbb{T}_{J}$ is as explained above.

Proof. This is proven by Lev73, Prop. 1] for $\mathrm{LCA}_{\mathcal{O}, c g}$. We follow his ideas, but give a shorter treatment by allowing ourselves to use the classical structure theorems for LCA L $_{c g}$ as in Mos67, Theorem 2.4] in full. (Step 1) Suppose $G \in \mathrm{LCA}_{\mathcal{O}, n s s}$. Then the underlying additive group has no small subgroups, so by Mos67, Theorem 2.4] there is an isomorphism of LCA groups

$$
G \simeq \mathbb{R}^{n} \oplus \mathbb{T}^{m} \oplus D \quad(\text { in LCA })
$$

for some $n, m \geq 0$ and $D$ discrete. By Lemma 2.11. we can promote the second direct sum in Equation 2.3, underlined in

$$
G \simeq\left[\mathbb{R}^{n} \oplus \mathbb{T}^{m}\right] \underline{\oplus D} \quad\left(\text { in } \mathrm{LCA}_{\mathcal{O}}\right)
$$

to a direct sum decomposition in the category $L C A_{\mathcal{O}}$. (Step 2) Now consider the object $\left[\mathbb{R}^{n} \oplus \mathbb{T}^{m}\right]$ in $\mathrm{LCA}_{\mathcal{O}}$. On the level of the underlying LCA group, its Pontryagin dual, just on the level of LCA, satisifes $\left[\mathbb{R}^{n} \oplus \mathbb{T}^{m}\right]^{\vee} \simeq \mathbb{R}^{n} \oplus \mathbb{Z}^{m}$ (in LCA). Run the same argument as before, Lemma 2.11 and so we can promote this isomorphism to

$$
\left[\mathbb{R}^{n} \oplus \mathbb{T}^{m}\right]^{\vee} \simeq \mathbb{R}^{n} \underline{\oplus} \mathbb{Z}^{m} \quad\left(\text { in } \mathrm{LCA}_{\mathcal{O}}\right),
$$

now again with the underlined direct sum on the level of LCA ${ }_{\mathcal{O}}$. Take Pontryagin duals again, this time in $\mathrm{LCA}_{\mathcal{O}}$, to get an isomorphism $\left[\mathbb{R}^{n} \oplus \mathbb{T}^{m}\right]^{\vee \vee} \cong \mathbb{R}^{n} \oplus \mathbb{T}^{m}$ in $\mathrm{LCA}_{\mathcal{O}}$. Combining these isomorphisms and using reflexivity of double dualization, we have managed to promote the isomorphism in Equation 2.3 from a direct sum in LCA to one in LCA $\mathcal{O}$. (Step 3) If instead $G \in \mathrm{LCA}_{\mathcal{O}, c g}$, use Proposition 2.6 to get $G^{\vee} \in \mathrm{LCA}_{\mathcal{O}, n s s}$, use the above structure theorem there and dualize again.

Lemma 2.14. Let $G \in \mathrm{LCA}_{\mathcal{O}}$ be given. Then there exists a clopen $\mathcal{O}$-submodule $H$ which is compactly generated.

Proof. If $G=0$ the claim is trivial. Thus, assume $G \neq 0$. Let $g \in G$ be a non-zero element. As $G$ is locally compact, we can find a compact neighbourhood $U$ of $g$. As $\mathcal{O}$ is finite over the integers, pick module generators so that $\mathcal{O}=\mathbb{Z}\left\langle\beta_{1}, \ldots, \beta_{n}\right\rangle$. Define

$$
U^{\prime}:=\bigcup_{i=1}^{n}\left(\beta_{i} U \cup-\beta_{i} U\right) .
$$

As a finite union of $2 n$ compact sets, $U^{\prime}$ is again compact, and clearly symmetric. Define $H:=\bigcup_{n \geq 1} U^{\prime n}$. We conclude that $H$ is compactly generated and clopen (cf. [Mor77, Prop. 8]). We claim that it is also an $\mathcal{O}$-submodule: For every $h \in H$, every $\left(\sum_{i=1}^{n} m_{i} \beta_{i}\right) h$ with $m_{i} \in \mathbb{Z}$ can be written as a finite sum of terms of the shape $\pm \beta_{i} h$ by writing the $m_{i}$ as repeated sums of \pm 1 .

Theorem 2.15 (Levin [Lev73, Theorem 2]). Suppose $G$ is a locally compact $\mathcal{O}$-module. Then there exists some vector $\mathcal{O}$-module $\bigoplus_{\sigma \in I} \mathbb{R}_{\sigma}$ (for some finite list I of real and complex places) and a compact $\mathcal{O}$-submodule $C$ such that

$$
\bigoplus_{\sigma \in I} \mathbb{R}_{\sigma} \oplus C \hookrightarrow G \rightarrow D
$$

is an exact sequence in $\mathrm{LCA}_{\mathcal{O}}$ and $D$ a discrete $\mathcal{O}$-module. 
For $\mathcal{O}=\mathbb{Z}$, this becomes the classical Principal Structure Theorem of LCA groups due to Pontryagin and van Kampen.

Proof. We give a very short proof: By Lemma 2.14 we can find an exact sequence $H^{\prime} \hookrightarrow G \rightarrow D^{\prime}$ in $\mathrm{LCA}_{\mathcal{O}}$ with $H^{\prime}$ compactly generated and $D^{\prime}$ discrete since $H$ is clopen [Mor77, Prop. 14]. Thus, $H^{\prime} \simeq \bigoplus_{\sigma \in I} \mathbb{R}_{\sigma} \oplus \bigoplus_{J \in \mathcal{I}} J \oplus C$ by Proposition 2.13, so since each $J$ is discrete, $\bigoplus_{\sigma \in I} \mathbb{R}_{\sigma} \oplus C$ is still an open and thus clopen submodule of $G$.

Next, we need to recall the structure of injective and projective objects in LCA $A_{\mathcal{O}}$. For LCA these results are due to Moskowitz Mos67, for LCA $\mathcal{O}$ due to Kryuchkov.

Theorem 2.16 (Kryuchkov, Kry97)). For an object $G \in \mathrm{LCA}_{\mathcal{O}}$ the following are equivalent:

(1) $G$ is an injective object.

(2) $G \simeq \bigoplus_{\sigma \in I} \mathbb{R}_{\sigma} \oplus \prod_{J \in \mathcal{I}} \mathbb{T}_{J}$, where $I$ is a finite list of real and complex places and $\mathcal{I}$ a (possibly infinite) list of ideals in $\mathcal{O}$.

(3) The additive group of $G$ is an injective object in LCA and the underlying algebraic $\mathcal{O}$-module is an injective object in $\mathcal{O}$-modules.

For the proof we refer to Kryuchkov [Kry97] or the Appendix

Theorem 2.17 (Kryuchkov, Kry97). For an object $G \in \mathrm{LCA}_{\mathcal{O}}$ the following are equivalent:

(1) $G$ is a projective object.

(2) $G \simeq \bigoplus_{\sigma \in I} \mathbb{R}_{\sigma} \oplus \prod_{J \in \mathcal{I}} J$, where $I$ is a finite list of real and complex places and $\mathcal{I}$ a (possibly infinite) list of ideals in $\mathcal{O}$.

(3) The additive group of $G$ is a projective object in LCA and the underlying algebraic $\mathcal{O}$ module is a projective object in $\mathcal{O}$-modules.

Proof. Take Pontryagin duals, use Theorem 2.16, and dualize again.

Corollary 2.18. An object $G \in \mathrm{LCA}_{\mathcal{O}}$ is simultaneously injective and projective if and only if it is isomorphic to $\bigoplus_{\sigma \in I} \mathbb{R}_{\sigma}$ for a finite list I of real and complex places.

Remark 2.19. As each $\mathbb{R}_{\sigma}$ is an injective object in $\mathrm{LCA}_{\mathcal{O}}$, they always split off as a direct summand. Thus, instead of the presentation of Theorem 2.15 we may write any object $G \in$ $\mathrm{LCA}_{\mathcal{O}}$ as $G \simeq \bigoplus_{\sigma \in I} \mathbb{R}_{\sigma} \oplus \tilde{G}$ for some $\tilde{G} \in \mathrm{LCA}_{\mathcal{O}}$ with $H \hookrightarrow G \rightarrow D$ exact, $H$ compact and $D$ discrete.

Theorem 2.20 (Kryuchkov, Kry97). We have the following:

(1) An object $G \in \mathrm{LCA}_{\mathcal{O}}$ has an injective resolution if and only if it lies in $\mathrm{LCA}_{\mathcal{O}, c g}$. In this case, it admits a length 2 injective resolution.

(2) An object $G \in \mathrm{LCA}_{\mathcal{O}}$ has a projective resolution if and only if it lies in $\mathrm{LCA}_{\mathcal{O}, n s s}$. In this case, it admits a length 2 projective resolution.

Proof. (1) Suppose $G$ has an injective resolution $G \rightarrow I^{0} \rightarrow I^{1} \rightarrow \cdots$. Then $G \hookrightarrow I^{0}$ is an admissible monic, but $I^{0} \simeq \bigoplus_{\sigma \in I} \mathbb{R}_{\sigma} \oplus \prod_{J \in \mathcal{I}} \mathbb{T}_{J}$ by the characterization of injectives, Theorem 2.16. As the tori $\mathbb{T}_{J}$ are compact and thus $\prod_{J \in \mathcal{I}} \mathbb{T}_{J}$ is compact by Tychonoff's Theorem, and then Proposition 2.13 implies that $I^{0}$ is compactly generated. However, closed subgroups of compactly generated LCA groups are themselves compactly generated, Mos67, Theorem 2.6], i.e. $G \in \mathrm{LCA}_{\mathcal{O}, c g}$. Conversely, suppose $G \in \mathrm{LCA}_{\mathcal{O}, c g}$. By Proposition 2.13 there exists an isomorphism

$$
G \simeq \bigoplus_{\sigma \in I} \mathbb{R}_{\sigma} \oplus \bigoplus_{J \in \mathcal{I}} J \oplus C
$$

in $\mathrm{LCA}_{\mathcal{O}}$ with $C$ a compact $\mathcal{O}$-module, $\mathcal{I}$ a finite list of ideals in $\mathcal{O}$, and $I$ a finite list of real and complex places. 
(Part A) The dual $C^{\vee}$ is a discrete $\mathcal{O}$-module. As $\mathcal{O}$ is a Dedekind domain, $C^{\vee}$ has an algebraic $\mathcal{O}$-module resolution of length 2 by projective algebraic $\mathcal{O}$-modules, $P_{1} \hookrightarrow P_{0} \rightarrow C^{\vee}$. This becomes an exact sequence in LCA $_{\mathcal{O}}$ tautologically since the topology is discrete, and indeed a projective resolution by Theorem 2.17 since the $P_{i}$ are algebraically projective and the underlying additive group is isomorphic to $\mathbb{Z}^{n}$ for a suitable $n$ and thus projective in LCA, Mos67, Theorem 3.3]. Dualize again, giving a length 2 injective resolution of $C$.

(Part B) For each ideal $J$ we have the Minkowski sequence

$$
J \hookrightarrow \bigoplus_{\sigma} \mathbb{R}_{\sigma} \rightarrow \mathbb{T}_{J}
$$

where $\sigma$ runs through all real and complex places once, and both types of terms $\mathbb{R}_{\sigma}$ resp. $\mathbb{T}_{J}$ are injective by Theorem 2.16. Thus, this is a length 2 injective resolution of $J$.

(Part C) By Theorem 2.16 the objects $\mathbb{R}_{\sigma}$ are already injectives.

Now take the direct sum of the exact sequences of Parts A, B, C for all summands appearing in Equation 2.4. As a finite direct sum of injectives, this remains an injective resolution, now of $G$.

(2) The discussion of the projective resolution follows from dualizing everything.

In particular, $\mathrm{LCA}_{\mathcal{O}}$ neither has enough injectives nor enough projectives, irrespective of the number field.

\section{Setting up a CAlculus of Fractions}

We now establish some factorization properties which are important to settle the issues mentioned in the introduction. In case they hold, they ensure the existence of a quotient exact category.

These results form the technical core of the paper.

Definition 3.1. Let $\mathrm{D}$ be an exact category.

(1) A full subcategory $\mathrm{C} \subset \mathrm{D}$ is called left special if for every $Z \in \mathrm{C}$ and every admissible epic $G \rightarrow Z$ in the category $\mathrm{D}$ there exists a commutative diagram

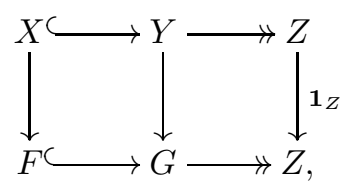

where the rows are exact, and the objects in the top row all lie in $\mathrm{C}$.

(2) A full subcategory $\mathrm{C} \subset \mathrm{D}$ is called left filtering if every morphism $G \rightarrow X$ in $\mathrm{D}$ such that $G \in \mathrm{C}$ admits a factorization

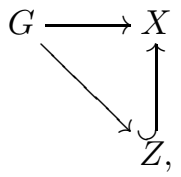

where $Z \in \mathrm{C}$.

We call $\mathrm{C} \subset \mathrm{D}$ right special (resp. right filtering) if $\mathrm{C}^{o p} \subset \mathrm{D}^{o p}$ is left special (resp. left special). We call $\mathrm{C} \subset \mathrm{D}$ left $s$-filtering, if it is left filtering and left special, resp. right s-filtering if $\mathrm{C}^{\mathrm{Op}} \subset \mathrm{D}^{\mathrm{op}}$ is left $s$-filtering.

We have given these definitions as in BGW16, §2.2]. These formulations are due to Bühler and are equivalent to the original concepts as introduced by Schlichting in Sch04. The equivalence of these formulations is proven in [BGW16, Appendix A]. 
Some very natural candidates for forming quotients do actually not satisfy Schlichting's conditions. This illustrates their subtle rôle:

Example 4. The full subcategory $\mathrm{LCA}_{C}$ is not left (or right) special in LCA. If it were left special, there would exist a compact group $C$ as in the left diagram below:
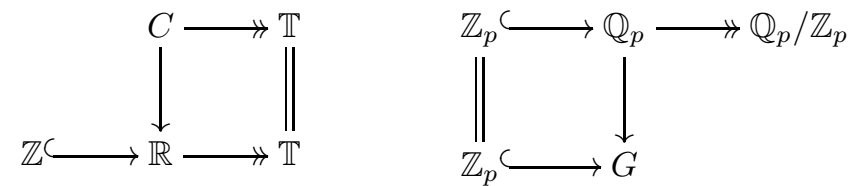

However, the image of $C$ in $\mathbb{R}$ necessarily is trivial, giving a contradiction. If it were right special, there would exist a compact group $G$ such that the diagram on the right commutes. The kernel of $\mathbb{Q}_{p} \rightarrow G$ is a closed subgroup and thus can only be $p^{i} \mathbb{Z}_{p}$ for some $i \in \mathbb{Z}$ (it cannot be trivial: If it were, $\mathbb{Q}_{p}$ would be a closed subgroup of a compact group and thus itself compact). However, these subgroups are even clopen. Thus, we get a factorization $\mathbb{Q}_{p} \rightarrow \mathbb{Q} / p^{i} \mathbb{Z}_{p} \rightarrow G$ with $\mathbb{Q} / p^{i} \mathbb{Z}_{p}$ is discrete. Hence, the image of the compact group $\mathbb{Z}_{p}$ in $\mathbb{Q} / p^{i} \mathbb{Z}_{p}$ is finite, and thus its image in $G$ is finite. This contradicts the injectivity of the map in the bottom row.

Example 5. Similarly, $\mathbf{L C A}_{\mathbb{R} C}$ is not left (or right) special in LCA. If it were left special, there would be a diagram

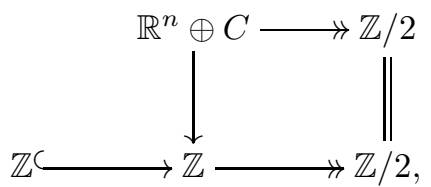

but the image of a connected or a compact group in $\mathbb{Z}$ must be zero. For right special, we can use the right side of Diagram 3.1 again.

Dually, these examples prove that $\mathrm{LCA}_{D}$ and $\mathrm{LCA}_{\mathbb{R} D}$ are neither left nor right special in LCA.

Let us move on to examples which do satisfy Schlichting's conditions. In fact, it turns out that it suffices to enlarge the categories of the previous examples slightly. Let $F$ be a number field and $\mathcal{O}$ its ring of integers.

Lemma 3.2. The full subcategory $\mathrm{LCA}_{\mathcal{O}, c g}$ is left filtering in $\mathrm{LCA}_{\mathcal{O}}$.

Proof. Let $f: G \rightarrow X$ be an arbitrary morphism in $\mathrm{LCA}_{\mathcal{O}}$ with $G$ compactly generated. It is clear that $f$ can be factored as

$$
G \stackrel{f}{\longrightarrow} \overline{\operatorname{im}_{\text {Set }}(f)} \hookrightarrow X,
$$

where $\overline{\text { im Set }_{\text {Se }}(f)}$ is the closure of the set-theoretic image of $f$ in the topology of $X$. By this choice, $\overline{\operatorname{im}_{\text {Set }}(f)} \hookrightarrow X$ is an admissible monic. This proves our claim once we will have shown that $\overline{\operatorname{im}_{\text {Set }}(f)}$ is compactly generated: As $G$ is compactly generated, there exists some symmetric compact subset $U \subseteq G$ such that $G=\bigcup_{n \geq 1} U^{n}$. Thus, $f(U)$ is compact and $\operatorname{im}_{\text {Set }}(f)=$ $\bigcup_{n \geq 1} f(U)^{n}$, so $f(U)$ is dense in $\overline{i_{\text {Set }}(f)}$. Secondly, $\overline{\operatorname{im}_{\text {Set }}(f)}$, as a closed subgroup of a locally compact group, is itself locally compact. Hence, we can find an open neighbourhood $V$ of zero such that $\bar{V}$ is compact. Thus, $\bar{V} \cup f(U)$ is compact and (in multiplicative notation)

$$
\overline{\operatorname{imset}_{\text {St }}(f)}=V \cdot\left(\bigcup_{n \geq 1} f(U)^{n}\right) \subseteq\left(\bigcup_{n \geq 1}(\bar{V} \cup f(U))^{n}\right) \subseteq \overline{\operatorname{im}_{\text {Set }}(f)}
$$

The equality on the left holds since, on its right side, one summand is open and the other dense. Hence, we have equality, and it follows that $\bar{V} \cup f(U)$ generates the closure. 
I have learnt this argument from an article of Fujita and Shakhmatov, which gives a detailed study of topological groups with a dense compactly generated subgroup [FS02, Theorem 2.7].

Lemma 3.3. The full subcategory $\mathrm{LCA}_{\mathcal{O}, n s s}$ is right filtering in $\mathrm{LCA}_{\mathcal{O}}$.

Proof. This is essentially dual to the previous argument; we give the details nonetheless: Let $f: X \rightarrow G$ be an arbitrary morphism in LCA $_{\mathcal{O}}$ such that $G$ has no small subgroups. Then $f^{\vee}: G^{\vee} \rightarrow X^{\vee}$ factors as

$$
G^{\vee} \rightarrow G^{\vee} / \operatorname{ker}\left(f^{\vee}\right) \longrightarrow \overline{\operatorname{im}_{\text {Set }}\left(f^{\vee}\right)} \hookrightarrow X^{\vee}
$$

and the middle arrow has dense set-theoretic image. As $G$ has no small subgroups, $G^{\vee}$ is compactly generated, cf. Proposition 2.6, then so is its admissible quotient $G^{\vee} / \operatorname{ker}\left(f^{\vee}\right)$ and thus $\overline{i_{\text {Set }}\left(f^{\vee}\right)}$ has a dense subgroup which is compactly generated. By the same argument as in the previous proof, $\overline{\operatorname{im}_{\text {Set }}\left(f^{\vee}\right)}$ is compactly generated, but

$$
(X / \operatorname{ker}(f))^{\vee} \cong \operatorname{ker}(f)^{\perp} \quad \text { and } \quad \operatorname{ker}(f)^{\perp} \cong \overline{\operatorname{imset~}_{\text {S }}\left(f^{\vee}\right)}
$$

(this is standard, but see [Nik98, Chapter $2, \S 9.1$ and $\S 9.2]$ for a review of such identities). Thus, $(X / \operatorname{ker}(f))^{\vee}$ is compactly generated, and therefore we obtain a factorization $X \rightarrow X / \operatorname{ker}(f) \rightarrow$ $G$, showing that $\mathrm{LCA}_{\mathcal{O}, n s s}$ is right filtering.

Lemma 3.4. The full subcategory $\mathrm{LCA}_{\mathcal{O}, c g}$ is left special in $\mathrm{LCA}_{\mathcal{O}}$.

Proof. (Step 1) We first show a more restrictive statement. Suppose

$$
G^{\prime \prime} \hookrightarrow G \rightarrow C
$$

is an exact sequence with $C$ compact. We apply the Structure Theorem to $G$, in the form of Remark 2.19, and get a diagram

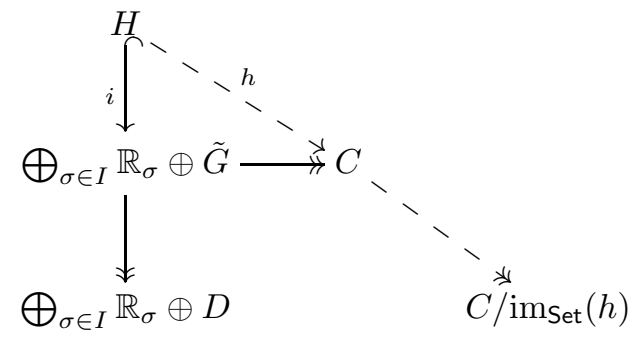

with $H$ compact, $D$ discrete. As $H$ is compact, the image of $h$ is compact, so the set-theoretic image $\operatorname{im}_{\text {Set }}(h)$ agrees with the categorical image, and is a closed subgroup of $C$. This justifies why the second dashed diagonal arrow in the above diagram is an admissible epic. By the universal property of cokernels we get a new diagram

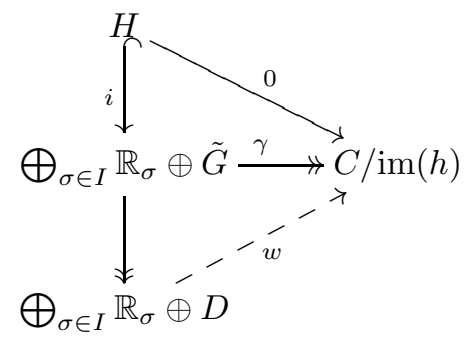

By [B̈̈h10, Cor. 7.7] the morphism $w$ must be an admissible epic. Let us, temporarily, just consider $w$ from the viewpoint of the underlying locally compact additive groups: Regarded in 
LCA, $w$ remains an admissible epic, and is of the shape

$$
w: \mathbb{R}^{n} \oplus D \rightarrow C / \operatorname{im}(h) .
$$

All admissible quotients of $\mathbb{R}^{n} \oplus D$ have the shape $\mathbb{R}^{i} \oplus \mathbb{T}^{j} \oplus D^{\prime}$ with $D^{\prime}$ discrete (this follows from the classification of the closed subgroups of $\mathbb{R}^{n} \oplus D$, see Corollary 2 to Theorem 7 in Mor77]). On the other hand, $C / \operatorname{im}(h)$ is compact, so its underlying additive topological group can only have the shape $\mathbb{T}^{j} \oplus D^{\prime}$ for $D^{\prime}$ finite. We return to LCA $\mathcal{O}$ : By the previous argument $C / \operatorname{im}(h)$ has no small subgroups, so by Theorem 2.20 it has a projective resolution. In fact, as the proof of the theorem loc. cit. explains, we find an admissible epic of the shape

$$
\beta_{0}: \bigoplus_{\nu \in I^{\prime}} \mathbb{R}_{\nu} \oplus \bigoplus_{J \in \mathcal{I}} J \rightarrow C / \operatorname{im}(h)
$$

for $I^{\prime}$ a finite list of real and complex places, while $\mathcal{I}$ is a finite list of ideals of $\mathcal{O}$, and the object on the left is projective in LCA $\mathcal{O}_{\mathcal{O}}$. The source being projective, this morphism $\beta_{0}$ can be lifted along the epic $\gamma$ to some morphism $\beta: \bigoplus_{\nu \in I^{\prime}} \mathbb{R}_{\nu} \oplus \bigoplus_{J \in \mathcal{I}} J \rightarrow \bigoplus_{\sigma \in I} \mathbb{R}_{\sigma} \oplus \tilde{G}$. Returning to Diagram 3.2 we now get

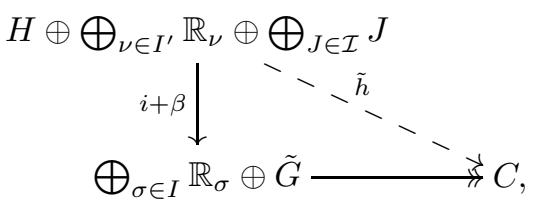

where $\tilde{h}$ is defined on $H$ by $\left.\tilde{h}\right|_{H}:=h$ (this refers to $h$ in Diagram [3.2), and on $\bigoplus_{\nu \in I^{\prime}} \mathbb{R}_{\nu} \oplus$ $\bigoplus_{J \in \mathcal{I}} J$ the map $\tilde{h}$ is defined by following the other arrows of the triangle. Finally, note that $\tilde{h}$ is, by construction, continuous and surjective, and since $I^{\prime}, \mathcal{I}$ are finite, its source is $\sigma$-compact, so by Pontryagin's Open Mapping Theorem the map $\tilde{h}$ is also open. It follows that $\tilde{h}$ is an admissible epic. Moreover, $H \oplus \bigoplus_{\nu \in I^{\prime}} \mathbb{R}_{\nu} \oplus \bigoplus_{J \in \mathcal{I}} J$ is compactly generated.

As a result, we obtain the commutative diagram

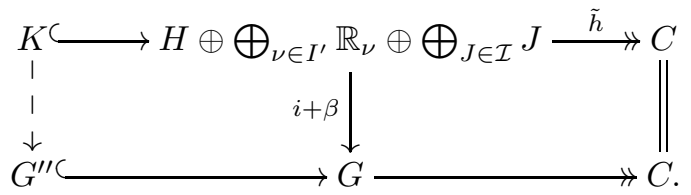

Here $K:=\operatorname{ker}(\tilde{h})$ and the dashed arrow exists by universal property. Finally, $K$ is also compactly generated, because it is a closed subgroup of a compactly generated group Mos67, Theorem 2.6]. It follows that the top row lies in $\mathrm{LCA}_{\mathcal{O}, c g}$. (Step 2) Now let

$$
G^{\prime \prime} \hookrightarrow \hat{G} \rightarrow G^{\prime}
$$

be an arbitrary exact sequence in $\mathrm{LCA}_{\mathcal{O}}$ with $G^{\prime}$ compactly generated. By Proposition 2.13 we have

$$
G^{\prime} \simeq P \oplus C \quad \text { with } \quad P:=\bigoplus_{\sigma \in I^{\prime}} \mathbb{R}_{\sigma} \oplus \bigoplus_{J \in \mathcal{I}^{\prime}} J
$$

with $C$ a compact $\mathcal{O}$-module, $\mathcal{I}^{\prime}$ a finite list of ideals in $\mathcal{O}$, and $I^{\prime}$ a finite list of real and complex places. The object $P$ is both compactly generated and projective by Theorem 2.17, Thus, it can be split off as a direct summand. Hence, we get an exact sequence

$$
G^{\prime \prime} \hookrightarrow G \rightarrow C
$$


for some complement $G$ of $P$ in $\hat{G}$, and the exact sequence in Equation 3.5 is isomorphic to $G^{\prime \prime} \hookrightarrow P \oplus G \rightarrow P \oplus C$. Apply Step 1 to Equation 3.6 i.e. we arrive at Diagram 3.4 Now, using the projectivity of $P$ and the resulting splittings, add the summand $P$ compatibly, giving

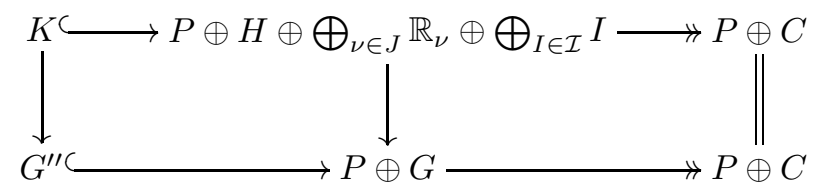

Again, all groups in the top row are compactly generated. We conclude that $\mathrm{LCA}_{\mathcal{O}, c g}$ is left special in $\mathrm{LCA}_{\mathcal{O}}$.

Lemma 3.5. The full subcategory $\mathrm{LCA}_{\mathcal{O}, \text { nss }}$ is right special in $\mathrm{LCA}_{\mathcal{O}}$.

Using Proposition 2.6, this is the Pontryagin dual statement to Lemma 3.4

We can obtain some further variations with no extra work:

Lemma 3.6. The full subcategory $\mathrm{LCA}_{\mathcal{O}, C}$ is left s-filtering in $\mathrm{LCA}_{\mathcal{O}, \mathbb{R} C}$.

Proof. We first prove left special: We use exactly the same proof as of Lemma 3.4 (we only need Step 1 this time), and since we now are in $\mathrm{LCA}_{\mathcal{O}, \mathbb{R} C}$, we may additionally assume $D=0$. So we must have $D^{\prime}=0$, and thus can run the argument with $\mathcal{I}=\varnothing$ in Equation 3.3 giving the claim. Left filtering is clear: Given any $f: G \rightarrow X$ with $G$ compact, it factors as $G \rightarrow \operatorname{im}_{\text {set }}(f) \rightarrow X$, but since $G$ is compact, its image $\operatorname{im}_{\text {Set }}(f)$ is also compact, thus closed, i.e. $\operatorname{im}_{\text {Set }}(f) \hookrightarrow X$ is an admissible monic.

Lemma 3.7. The full subcategory $\mathrm{LCA}_{\mathcal{O}, D}$ is right s-filtering in $\mathrm{LCA}_{\mathcal{O}, \mathbb{R} D}$.

Again, this is just the Pontryagin dual statement.

Lemma 3.8. The category $\mathrm{LCA}_{\mathcal{O}, \mathbb{R} D}$ is extension-closed in $\mathrm{LCA}_{\mathcal{O}}$.

We begin by proving a special case of the claim in LCA, i.e. for $\mathcal{O}=\mathbb{Z}$ :

Lemma 3.9. Suppose $G^{\prime} \hookrightarrow G \rightarrow N$ is an exact sequence in LCA with $G^{\prime} \in \mathrm{LCA}_{\mathbb{R} D}$ and $N$ discrete, then $G \in \mathrm{LCA}_{\mathbb{R} D}$.

Proof. Consider the extension $\mathbb{R}^{i} \oplus D^{\prime} \hookrightarrow G \rightarrow N$ with $D^{\prime}$ discrete. We get the filtration $\mathbb{R}^{i} \hookrightarrow \mathbb{R}^{i} \oplus D^{\prime} \hookrightarrow G$. The variant of Noether's Lemma for exact categories Büh10, Lemma $3.5]$, yields the exact sequence $\left(\mathbb{R}^{i} \oplus D^{\prime}\right) / \mathbb{R}^{i} \hookrightarrow G / \mathbb{R}^{i} \rightarrow G /\left(\mathbb{R}^{i} \oplus D^{\prime}\right)$ and this simplifies to $D^{\prime} \hookrightarrow G / \mathbb{R}^{i} \rightarrow N$. As $D^{\prime}$ and $N$ are discrete and discrete groups are closed under extension, it follows that we have an exact sequence $\mathbb{R}^{i} \hookrightarrow G \rightarrow D$ with $D$ discrete. However, $\mathbb{R}^{i}$ is an injective object and thus this sequence must split. Hence, $G \simeq \mathbb{R}^{i} \oplus D$ with $D$ discrete, giving the claim.

Proof of Lemma 3.8. (Step 1) First, we prove this for $\mathcal{O}=\mathbb{Z}$, i.e. in LCA. This is tedious to spell out, but quite easy: Let $\mathbb{R}^{i} \oplus D^{\prime} \hookrightarrow G \rightarrow \mathbb{R}^{j} \oplus D^{\prime \prime}$ be an extension with $D^{\prime}, D^{\prime \prime}$ discrete groups. By the Structure Theorem (Theorem 2.15), $G$ decomposes as $\mathbb{R}^{n} \oplus H \hookrightarrow G \rightarrow D, H$ compact, $D$ discrete. Consider the composition $h: H \hookrightarrow G \rightarrow \mathbb{R}^{j} \oplus D^{\prime \prime}$. As $H$ is compact, the image of $H$ after projecting to $\mathbb{R}^{j}$ is compact and thus trivial. Hence, the map factors as $H \rightarrow D^{\prime \prime}$, and since $H$ is compact but $D^{\prime \prime}$ discrete, the image imset $(h)$ must be finite. Thus, we 
get a factorization

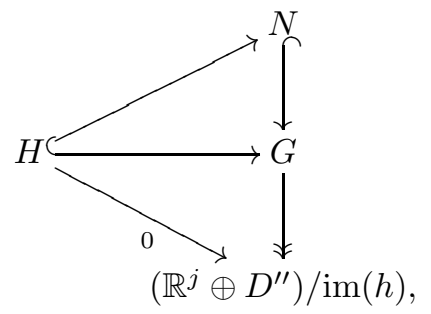

where $N$ is defined as the kernel of the downward admissible epic originating from $G$. As this quotients out more from $G$, we get a filtration $\mathbb{R}^{i} \oplus D^{\prime} \hookrightarrow N \hookrightarrow G$ and by Noether's Lemma, the corresponding sequence of quotients $\frac{N}{\mathbb{R}^{i} \oplus D^{\prime}} \hookrightarrow \frac{G}{\mathbb{R}^{i} \oplus D^{\prime}} \rightarrow \frac{G}{N}$ is exact. As $G / N \simeq$ $\left(\mathbb{R}^{j} \oplus D^{\prime \prime}\right) / \operatorname{im}_{\text {Set }}(h)$ by the choice of $N$, and $G /\left(\mathbb{R}^{i} \oplus D^{\prime}\right) \simeq \mathbb{R}^{j} \oplus D^{\prime \prime}$, we learn that $N /\left(\mathbb{R}^{i} \oplus D^{\prime}\right) \simeq$ $\operatorname{im}_{\text {Set }}(h)$. Hence, $\mathbb{R}^{i} \oplus D^{\prime} \hookrightarrow N \rightarrow \operatorname{im}_{\text {Set }}(h)$ is exact. It follows that $N$ is an extension of a group in $\mathrm{LCA}_{\mathbb{R} D}$ by a finite group, i.e. $N \simeq \mathbb{R}^{i} \oplus E$ with $E$ discrete by Lemma 3.9. In Diagram 3.7 the factorization $H \rightarrow N$ is an admissible monic by Büh10, Corollary 7.7] and thus it becomes $H \hookrightarrow \mathbb{R}^{i} \oplus E$. As $H$ is compact and $\mathbb{R}^{i}$ has no non-trivial compact subgroups, $H$ must lie in $E$, but the latter is discrete, so $H$ must be a finite group. Thus, we have $\mathbb{R}^{n} \oplus H \hookrightarrow G \rightarrow D$ with $D$ discrete and $H$ finite. Now Lemma 3.9 implies that $G$ lies in LCA $_{\mathbb{R} D}$. (Step 2) Finally, the case of general $\mathcal{O}$ reduces to the case over $\mathbb{Z}$ by Lemma 2.11

Example 6. The morphism $\mathbb{Z} \rightarrow \mathbb{R}$ is an element of $\operatorname{Hom}(\mathbb{Z}, \mathbb{R})$, both in $L C A$ as well as in $L C A_{\mathbb{R} D}$. It is an admissible monic in LCA, but not in $\mathrm{LCA}_{\mathbb{R} D}$ as the quotient $\mathbb{T}$ does not lie in $\mathrm{LCA}_{\mathbb{R} D}$.

Lemma 3.10. The category $\mathrm{LCA}_{\mathcal{O}, \mathbb{R} C}$ is extension-closed in $\mathrm{LCA}_{\mathcal{O}}$.

Corollary 3.11. All the full subcategories $\mathrm{LCA}_{\mathcal{O}, C}, \mathrm{LCA}_{\mathcal{O}, D}, \mathrm{LCA}_{\mathcal{O}, \mathbb{R} D}, \mathrm{LCA}_{\mathcal{O}, \mathbb{R} C}, \mathrm{LCA}_{\mathcal{O}, c g}$, $\mathrm{LCA}_{\mathcal{O}, n s s}$ are fully exact subcategories of $\mathrm{LCA}_{\mathcal{O}}$. In particular, they all carry a canonical exact structure and are closed under extensions in $\mathrm{LCA}_{\mathcal{O}}$. A sequence is exact in these categories if and only if it is exact in LCA; that is: The forgetful functor

$$
\mathrm{LCA}_{\mathcal{O},(-)} \longrightarrow \mathrm{LCA}
$$

is faithful, exact and reflects exactness.

Proof. For this type of claim, thanks to Büh10, Lemma 10.20] we only need to know that the full subcategory in question is closed under extensions. For LCA LC $_{c g}, \mathrm{LCA}_{n s s}$ this was shown by Moskowitz, see Proposition 2.6 (we could take an alternative route here: By BGW16, Lemma 2.14] left special subcategories are closed under extensions. Dually, the same is true for right

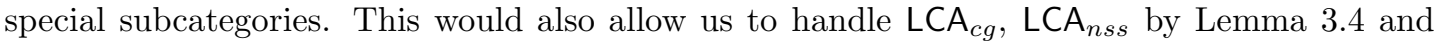
Lemma 3.5). The claims for $\mathrm{LCA}_{\mathcal{O}, c g}, \mathrm{LCA}_{\mathcal{O}, n s s}$ follow from this as they refer to properties which only depend on the underlying additive group. For LCA $\mathrm{O}_{\mathcal{D}}$ in LCA note that any extension of discrete $\mathcal{O}$-modules is discrete, so it is extension-closed in LCA $\mathcal{O}$. Taking the Pontryagin dual statement, we get that $\mathrm{LCA}_{\mathcal{O}, C}$ is closed under extensions. For $\mathrm{LCA}_{\mathcal{O}, \mathbb{R} D}, \mathrm{LCA}_{\mathcal{O}, \mathbb{R} C}$ we had already shown that they are closed under extensions (Lemma 3.8. Lemma 3.10). The functor $\mathrm{LCA}_{\mathcal{O},(-)} \longrightarrow \mathrm{LCA}$ is exact and reflects exactness as a corollary of the exact structures on all these categories being defined through [Büh10, Lemma 10.20]. The faithfulness is clear.

\section{Proof of the MAIN THEOREM}

Let us quickly recall how exact categories and the notions of left (resp. right) $s$-filtering are connected to stable $\infty$-categories. Firstly, Schlichting has shown that the natural notion of an exact sequence of exact categories induces an exact sequence of homotopy categories. 
Theorem 4.1 (Schlichting Localization). Let $\mathrm{C}$ be an idempotent complete exact category and $\mathrm{C} \hookrightarrow \mathrm{D}$ a right (or left) s-filtering inclusion into an exact category $\mathrm{D}$. Then

$$
D^{b}(\mathrm{C}) \hookrightarrow D^{b}(\mathrm{D}) \rightarrow D^{b}(\mathrm{D} / \mathrm{C})
$$

is an exact sequence of triangulated categories.

This is [Sch04, Prop. 2.6]. See Keller [Kel96, §11] or Bühler [Büh10, §10] for the construction of the category of chain complexes and the homotopy category. Secondly, exactness on the homotopy category level is essentially equivalent to all other conceivable notions of exactness, see the discussion in [BGT13, §5].

Next, recall the Eilenberg swindle. Although absolutely classical, we record it because it is so crucial for the computation:

Lemma 4.2. If an exact category $\mathrm{C}$ is closed under countable products or coproducts and $K: \mathrm{Cat}_{\infty}^{\mathrm{ex}} \rightarrow \mathrm{A}$ is any additive invariant (e.g., a localizing invariant), then $K(\mathrm{C})=0$.

Proof. Suppose C has countable coproducts. The Eilenberg swindle functor is defined by

$$
E: \mathrm{C} \longrightarrow \mathcal{E} \mathrm{C}, \quad X \mapsto\left(X \hookrightarrow \coprod_{i \in \mathbb{Z}} X \rightarrow \coprod_{i \in \mathbb{Z}} X\right),
$$

where $\mathcal{E} A b$ denotes the exact category of exact sequences and the epic is the 'shift one to the left' functor. Projecting to the left, middle and right term of $\mathcal{E C}$, call these $p_{i}: \mathrm{C} \rightarrow \mathrm{C}$ with $i=0,1,2$, the additivity of $K$ implies that $p_{1}=p_{0}+p_{2}$ holds for the maps induced on $K(\mathrm{C}) \rightarrow K(\mathrm{C})$, but $p_{1}=p_{2}$, so clearly $p_{0}=0$. However, $p_{0}$ is just the identity functor. If $\mathrm{C}$ has countable products, perform the same trick with products instead.

Lemma 4.3. For every localizing invariant $K: \mathrm{Cat}_{\infty}^{\mathrm{ex}} \rightarrow \mathrm{A}$, there are canonical equivalences

$$
K\left(\mathrm{LCA}_{\mathcal{O}, n s s}\right) \stackrel{\sim}{\longrightarrow} K\left(\mathrm{LCA}_{\mathcal{O}, \mathbb{R}}\right) \quad \text { and } \quad K\left(\mathrm{LCA}_{\mathcal{O}, c g}\right) \stackrel{\sim}{\longrightarrow} K\left(\mathrm{LCA}_{\mathcal{O}, \mathbb{R}}\right) .
$$

Taking Pontryagin duals on the left and vector space duals on the right transforms one equivalence into the other.

Proof. (Step 1) Firstly, we establish equivalences

$$
K\left(\mathrm{LCA}_{\mathcal{O}, \mathbb{R} D}\right) \stackrel{\sim}{\longrightarrow} K\left(\mathrm{LCA}_{\mathcal{O}, n s s}\right) \quad \text { and } \quad K\left(\mathrm{LCA}_{\mathcal{O}, \mathbb{R} C}\right) \stackrel{\sim}{\longrightarrow} K\left(\mathrm{LCA}_{\mathcal{O}, c g}\right) .
$$

Consider $\mathrm{LCA}_{\mathcal{O}, n s s}$ : These modules admit a projective resolution, see Theorem 2.20. The resolution then has the shape $P_{1} \hookrightarrow P_{0} \rightarrow G$. By the classification of projectives in LCA $_{\mathcal{O}}$, Theorem 2.17, for both $i=0,1$ we have an isomorphism

$$
P_{i} \simeq \bigoplus_{\sigma \in I_{i}} \mathbb{R}_{\sigma} \oplus \prod_{J \in \mathcal{I}_{i}} J,
$$

where $I_{i}$ is a finite list of real and complex places and $\mathcal{I}_{i}$ a (possibly infinite) list of ideals in $\mathcal{O}$. In particular, every object in $\mathrm{LCA}_{\mathcal{O}, n s s}$ has a two-term resolution by projectives in $\mathrm{LCA}_{\mathcal{O}, \mathbb{R} D}$. The claim for $\mathrm{LCA}_{\mathcal{O}, c g}$ is the Pontryagin dual statement.

(Step 2) Lemma 3.7 shows that $\mathrm{LCA}_{\mathcal{O}, D} \hookrightarrow \mathrm{LCA}_{\mathcal{O}, \mathbb{R} D}$ is right $s$-filtering. Thus, the quotient exact category exists and it is easy to see that the natural functor

$$
\mathrm{LCA}_{\mathcal{O}, \mathbb{R}} \stackrel{\sim}{\longrightarrow} \mathrm{LCA}_{\mathcal{O}, \mathbb{R} D} / \mathrm{LCA}_{\mathcal{O}, D}
$$

is an exact equivalence of exact categories (it is fully faithful, clearly exact and essentially surjective). Now, by Schlichting's Localization Theorem, $K\left(\mathrm{LCA}_{\mathcal{O}, D}\right) \rightarrow K\left(\mathrm{LCA}_{\mathcal{O}, \mathbb{R} D}\right) \rightarrow K\left(\mathrm{LCA}_{\mathcal{O}, \mathbb{R}}\right)$ is a fiber sequence, but by the Eilenberg swindle, the left term is zero, Lemma 4.2, Finally, dualization of the entire argument handles $\mathrm{LCA}_{\mathcal{O}, c g}$. 
As $\mathrm{LCA}_{\mathcal{O}, c g} \hookrightarrow \mathrm{LCA}_{\mathcal{O}}$ is left filtering and left special, we get an exact sequence of exact categories

$$
\mathrm{LCA}_{\mathcal{O}, c g} \hookrightarrow \mathrm{LCA}_{\mathcal{O}} \rightarrow \mathrm{LCA}_{\mathcal{O}} / \mathrm{LCA}_{\mathcal{O}, c g}
$$

In particular, the quotient $\mathrm{LCA}_{\mathcal{O}} / \mathrm{LCA}_{\mathcal{O}, c g}$ exists in the 2-category of exact categories. Next, let $\operatorname{Mod}(\mathcal{O})$ be the category of all (algebraic) $\mathcal{O}$-modules, and $\operatorname{Mod}_{f g}(\mathcal{O})$ the subcategory of finitely generated $\mathcal{O}$-modules. Note that $\operatorname{Mod}_{f g}(\mathcal{O}) \hookrightarrow \operatorname{Mod}(\mathcal{O})$ is also left filtering and left special 1 . We obtain an exact sequence of abelian categories, and indeed get a commutative diagram

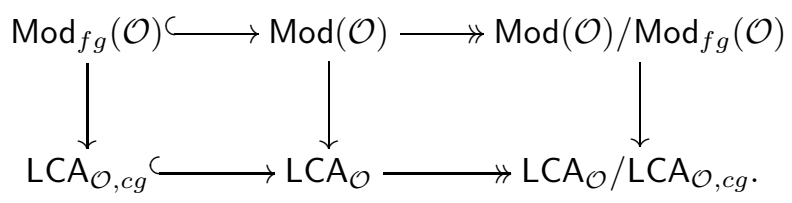

The downward arrow just sends an algebraic $\mathcal{O}$-module to its topological counterpart, equipped with the discrete topology. All arrows are exact functors.

Lemma 4.4. The functor $\operatorname{Mod}(\mathcal{O}) / \operatorname{Mod}_{f g}(\mathcal{O}) \longrightarrow \mathrm{LCA}_{\mathcal{O}} / \mathrm{LCA}_{\mathcal{O}, c g}$ is an exact equivalence of exact categories. Both categories are abelian.

Proof. The functor is essentially surjective: Given any $G$ in LCA $_{\mathcal{O}}$, the Structure Theorem (Theorem 2.15) presents it as

$$
\bigoplus_{\sigma \in I} \mathbb{R}_{\sigma} \oplus C \hookrightarrow G \rightarrow D
$$

with $C$ compact, $D$ discrete and $I$ a finite list. As $\bigoplus_{\sigma \in I} \mathbb{R}_{\sigma} \oplus C$ is compactly generated, $G \rightarrow D$ becomes an isomorphism in $\mathrm{LCA}_{\mathcal{O}} / \mathrm{LCA}_{\mathcal{O}, c g}$. As all modules in the essential image carry the discrete topology, there is no difference between arbitrary module homomorphisms or continuous ones, so the functor is moreover fully faithful and exact. As $\operatorname{Mod}(\mathcal{O}) / \operatorname{Mod}_{f g}(\mathcal{O})$ stems from a quotient of abelian categories, it is itself abelian (note that we quotient by a Serre subcategory). By the equivalence of categories, the same holds for $\mathrm{LCA}_{\mathcal{O}} / \mathrm{LCA} \mathrm{O}_{\mathcal{O}, c g}$.

We are ready to prove our main result.

Theorem 4.5. Let $F$ be a number field and $\mathcal{O}$ its ring of integers. For every localizing invariant $K: \mathrm{Cat}_{\infty}^{\mathrm{ex}} \rightarrow \mathrm{A}$, there is a canonical fiber sequence

$$
K(\mathcal{O}) \longrightarrow K(\mathbb{R})^{r} \oplus K(\mathbb{C})^{s} \longrightarrow K\left(\mathrm{LCA}_{\mathcal{O}}\right),
$$

where $r$ is the number of real places and $s$ the number of complex places.

Proof. By Schlichting's Localization Theorem, Theorem 4.1, both exact rows in Diagram 4.1 induce fiber sequences in any localizing theory,

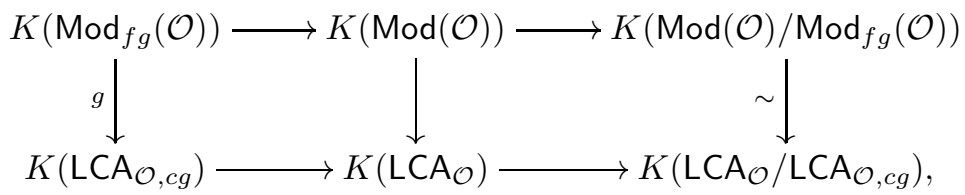

and the equivalence on the right stems from Lemma 4.4. Thus, the left square is a bi-cartesian square in $A$. As the category $\operatorname{Mod}(\mathcal{O})$ has countable coproducts, the Eilenberg swindle yields $K(\operatorname{Mod}(\mathcal{O}))=0$, see Lemma 4.2. Moreover, all objects in $\operatorname{Mod}_{f g}(\mathcal{O})$ have a short projective

\footnotetext{
${ }^{1}$ It is a Serre subcategory of an abelian category, so it is automatically left and right filtering. Left specialness is easy to check.
} 
resolution by the projective object $\mathcal{O}$, so $K\left(\operatorname{Mod}_{f g}(\mathcal{O})\right) \simeq K(\mathcal{O})$. By Lemma4.3, $K\left(\mathrm{LCA}_{\mathcal{O}, c g}\right) \cong$ $K\left(\mathrm{LCA}_{\mathcal{O}, \mathbb{R}}\right)$, and by Corollary 2.9 we have $K\left(\mathrm{LCA}_{\mathcal{O}, \mathbb{R}}\right) \cong K(\mathbb{R})^{r} \oplus K(\mathbb{C})^{s}$. Thus,

$$
K(\mathcal{O}) \longrightarrow K(\mathbb{R})^{r} \oplus K(\mathbb{C})^{s} \longrightarrow K\left(\mathrm{LCA}_{\mathcal{O}}\right)
$$

is a fiber sequence in $A$, proving the claim.

Finally, we identify the map:

Theorem 4.6. We keep the assumptions of the previous theorem. The map $K(\mathcal{O}) \longrightarrow K(\mathbb{R})^{r} \oplus$ $K(\mathbb{C})^{s}$ in our construction of Equation 4.2 is induced from the Minkowski embedding ring homomorphism $\mathcal{O} \rightarrow \mathbb{R}^{r} \oplus \mathbb{C}^{s}$.

Proof. Inspecting Diagram 4.1, the exact functor underlying the map $K(\mathcal{O}) \longrightarrow K(\mathbb{R})^{r} \oplus K(\mathbb{C})^{s}$ sends the object $\mathcal{O}$ of $\operatorname{Mod}_{f g}(\mathcal{O})$ to $\mathcal{O}$ in $\mathrm{LCA}_{\mathcal{O}, c g}$. This looks a priori different from the functor we want, however, both induce the same map on the level of $K$ : Let $\operatorname{Proj}\left(\operatorname{Mod}_{f g}(\mathcal{O})\right)$ denote the fully exact subcategory of $\operatorname{Mod}_{f g}(\mathcal{O})$ of projectives, generated by the single object $\mathcal{O}$. Define an exact functor $F: \operatorname{Proj}\left(\operatorname{Mod}_{f g}(\mathcal{O})\right) \rightarrow \mathcal{E} \mathrm{LCA}_{\mathcal{O}, c g}$, where the latter is the exact category of exact sequences, by its action on the generator:

$$
\mathcal{O} \mapsto\left(\mathcal{O} \hookrightarrow \bigoplus_{\sigma \in S} \mathbb{R}_{\sigma} \rightarrow \mathbb{T}_{\mathcal{O}}\right)
$$

That is: We send $\mathcal{O}$ to the Minkowski sequence of $\mathcal{O}$, cf. Equation 2.1] If

$$
p_{i}: \operatorname{Proj}\left(\operatorname{Mod}_{f g}(\mathcal{O})\right) \rightarrow \mathrm{LCA}_{\mathcal{O}, c g}
$$

for $i=0,1,2$ denote the projections to left, middle and right term, the additivity of $K$ implies that $p_{1}=p_{0}+p_{2}$ for the induced maps on $K$. However, $p_{0}$ induces the map $g$ in Diagram 4.3, while $p_{1}$ is the functor of our claim. Finally, the functor $p_{2}$ can be factored over the category of compact modules,

$$
p_{2}: \operatorname{Proj}\left(\operatorname{Mod}_{f g}(\mathcal{O})\right) \longrightarrow \mathrm{LCA}_{\mathcal{O}, C} \longrightarrow \mathrm{LCA}_{\mathcal{O}, c g} .
$$

Induced on $K$, this is $K\left(\operatorname{Mod}_{f g}(\mathcal{O})\right) \rightarrow 0 \rightarrow K\left(\mathrm{LCA}_{\mathcal{O}, c g}\right)$ by the Eilenberg swindle, Lemma 4.2 , Thus, $p_{2}$ induces the zero map on the level of $K$. It follows that $p_{0}$ and $p_{1}$ induce the same map on $K$. $3.4]$ :

In the special case of $\mathcal{O}=\mathbb{Z}$, we recover the statement of Clausen's theorem Cla17, Theorem

Theorem 4.7 (Clausen). For every localizing invariant $K: \mathrm{Cat}_{\infty}^{\mathrm{ex}} \rightarrow \mathrm{A}$, there is a canonical fiber sequence

$$
K(\mathbb{Z}) \longrightarrow K(\mathbb{R}) \longrightarrow K(\text { LCA }) .
$$

While this is the same claim as in Clausen's work, it does not allow us to conclude that we actually construct the same fiber sequence. This question remains open.

\section{Regulators}

In this section, $K$ really stands for $K$-theory. Let $F$ be a number field with $r$ real places and $s$ complex places and $\mathcal{O}$ its ring of integers. While this paper is about the computation of $K\left(\mathrm{LCA}_{\mathcal{O}}\right)$, the Minkowski map

$$
K(\mathcal{O}) \longrightarrow K(\mathbb{R})^{r} \oplus K(\mathbb{C})^{s}
$$

has, uncalled for, showed up in this computation.

Although there is a priori not much reason to expect regulators to play a rôle in the context of the category $L C A_{\mathcal{O}}$, it is noteworthy that the Borel regulator factors over the above map. This suggests that some "shadow" of the Borel regulator may be definable on the $K$-theory 
of $L C A_{\mathcal{O}}$. This turns out to be virtually true, but the image of the map becomes the torus which arises from quotienting out the regulator lattice from the target cohomology group; so it surjects onto the torus whose natural volume is precisely what is customarily called the $n$-th Borel regulator value, and which up to a rational factor is a regularized zeta value,

$$
\sim_{\mathbb{Q}^{\times}} \zeta_{F}^{\star}(1-n)
$$

As a side application of this, we obtain that the sequence in Theorem 4.5 splits into short exact sequences rationally.

Let us briefly recall the concept of the Borel regulator. The Dirichlet regulator map

$$
\Phi_{\text {Dirichlet }}: \mathcal{O}^{\times} \oplus \mathbb{Z} \longrightarrow \mathbb{R}^{r} \oplus \mathbb{R}^{s}
$$

sends a unit $x \in \mathcal{O}^{\times}$to the vector $(\log |\sigma(x)|)_{\sigma}$, where $\sigma$ runs through all real places and one representative of each complex place, and $\mathbb{Z}$ gets mapped to the right diagonally. The image of $\Phi_{\text {Dirichlet }}$ is a full rank lattice and the kernel a finite group, namely the roots of unity in $F^{\times}$. The covolume of the lattice is known as the Dirichlet regulator value itself.

In view of $K_{1}(\mathcal{O}) \cong \mathcal{O}^{\times}$, one may rephrase this map as a map defined on $K$-theory. Borel has given a far-reaching generalization of this map. Suppose $n \geq 1$. Conceptually, following Beilinson's picture, the Borel regulator is a canonical morphism

$$
\Phi_{\text {Borel }}: K_{2 n-1}(\mathcal{O}) \longrightarrow H_{\mathcal{D}}^{1}\left((\operatorname{Spec} F)_{/ \mathbb{R}}, \mathbb{R}(n)\right),
$$

but in the case at hand the Beilinson-Deligne cohomology group on the right is just a few copies of the real numbers, specifically

$$
\Phi_{\text {Borel }}: K_{2 n-1}(\mathcal{O}) \longrightarrow \begin{cases}\mathbb{R}^{s} & \text { if } n \text { is even } \\ \mathbb{R}^{r+s} & \text { if } n \text { is odd. }\end{cases}
$$

We write $\Phi_{\text {Borel }}: K_{2 n-1}(\mathcal{O}) \rightarrow \mathbb{R}^{d_{n}}$ with $d_{n}$ defined accordingly for simplicity.

Theorem 5.1 (Borel). For $n \geq 2$, the image of $\Phi_{\text {Borel }}$ is a full rank $\mathbb{Z}$-lattice and $\operatorname{ker}\left(\Phi_{\text {Borel }}\right)$ is a finite group. The covolume of the lattice satisfies

$$
u_{n} \cdot \lim _{z \rightarrow 1-n}(z-1+n)^{-d_{n}} \cdot \zeta_{F}(z),
$$

where $u_{n}$ is a non-zero rational number.

See Rap88, BG02 for details.

Remark 5.2. Instead, for $n=1$, one can use Dirichlet's analytic class number formula, giving an analogous statement. Note the slight difference in that Dirichlet regulator as in Equation 5.1 needs the additional artificial summand $\mathbb{Z}$ to have a full rank lattice image. This has no counterpart for Borel's regulator. There are some other subtle differences in degree one. For example $K_{1}(\mathcal{O})=\mathcal{O}^{\times}$and $K_{1}(F)=F^{\times}$are very different, while $K_{2 n-1}(\mathcal{O}) \cong K_{2 n-1}(F)$ for all $n \geq 2$.

Remark 5.3. For $n \geq 1$, the $K$-groups $K_{2 n}(\mathcal{O})$ are finite, so we cannot have non-trivial maps to the reals and thus not expect anything like a regulator.

We are now ready to prove the following:

Theorem 5.4. Let $F$ be a number field and $\mathcal{O}$ its ring of integers. With rational coefficients, the long exact sequence of Theorem 4.5 splits into short exact sequences

$$
0 \rightarrow K_{n}(\mathcal{O})_{\mathbb{Q}} \rightarrow K_{n}(\mathbb{R})_{\mathbb{Q}}^{r} \oplus K_{n}(\mathbb{C})_{\mathbb{Q}}^{s} \rightarrow K_{n}\left(\mathrm{LCA}_{\mathcal{O}}\right)_{\mathbb{Q}} \rightarrow 0
$$

for all $n$.

Note that for $n \geq 2$ and $n$ even, $K_{n}(\mathcal{O})_{\mathbb{Q}}=0$. 
Proof. We apply the fiber sequence of Theorem 4.5 for non-connective $K$-theory $\mathbb{K}$. As the ring $\mathcal{O}$ and fields (here $\mathbb{R}$ and $\mathbb{C}$ ) are regular, connective and non-connective $K$-theory agree for the first two terms in the fiber sequence. Thus, the resulting long exact sequence also holds for connective $K$-theory. We display it below in the bottom row:

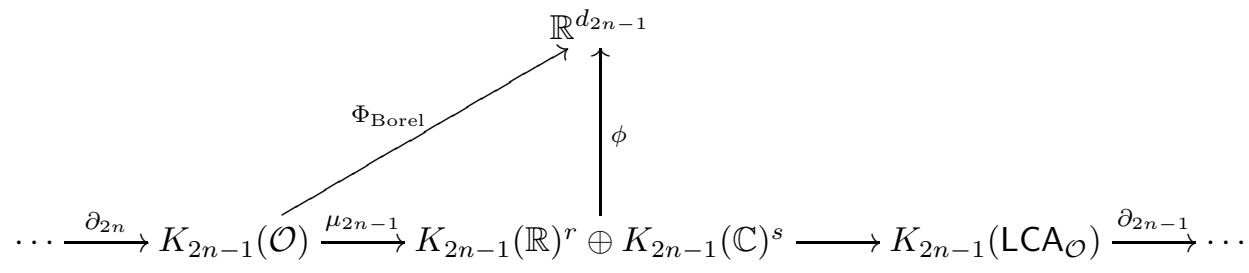

Next, note that for $n \geq 2$ the Borel regulator is defined as the composition of two maps: Firstly, a Minkowski type map

$$
K_{2 n-1}(\mathcal{O}) \stackrel{\sim}{\longrightarrow} K_{2 n-1}(F) \stackrel{M}{\longrightarrow} K_{2 n-1}(\mathbb{R})^{r} \oplus K_{2 n-1}(\mathbb{C})^{s} \longrightarrow\left[\bigoplus_{\sigma} K_{2 n-1}(\mathbb{C})\right]^{G},
$$

where $\sigma$ runs through all complex embeddings, and $[-]^{G}$ denotes taking $G$-fixed points under the complex conjugation involution, $M$ is induced from the Minkowski embedding as a ring homomorphism. The left-most map is induced from the inclusion $\mathcal{O} \hookrightarrow F$; this is an isomorphism on $K$-theory [Wei13, Ch. V, $\S 6$, Theorem 6.8]. Secondly, the regulator map defined on the level of $\mathbb{C}$, namely

$$
K_{2 n-1}(\mathbb{C}) \stackrel{\text { Hur }}{\longrightarrow} H_{2 n-1}\left(\left(B \mathrm{GL}(\mathbb{C})_{\delta}\right)^{+}, \mathbb{Z}\right) \stackrel{\sim}{\longrightarrow} H_{2 n-1}\left(B \mathrm{GL}(F)_{\delta}, \mathbb{Z}\right) \longrightarrow \mathbb{R}^{d_{n}},
$$

where the first map is the Hurewicz morphism, originating from Quillen's model for $K$-theory via the plus construction, $B \mathrm{GL}(\mathbb{C})_{\delta}$ denotes the classifying space of $\mathrm{GL}(\mathbb{C})_{\delta}$ as a group with the discrete topology; the second map comes from the invariance of group homology under the plus construction, and the last arrow is the pairing with the Borel class (this is induced from the continuous group cohomology of $\mathrm{GL}(\mathbb{C})$, pulled back along the tautologically continuous map $\mathrm{GL}(\mathbb{C})_{\delta} \rightarrow \mathrm{GL}(\mathbb{C})$, see $\left[\right.$ Rap88, BG02]). The composition of these maps is $\Phi_{\text {Borel }}$ in the above diagram. As we can see from Equation 5.4, up until the Minkowski map, the first ingredients in this compositions of maps agrees with the map appearing in the bottom sequence in Figure 5.3. Thus, if we denote the remaining steps by $\phi$, we get the middle upward arrow in the diagram. Next, note that

$$
\operatorname{ker}\left(\mu_{2 n-1}\right) \subseteq \operatorname{ker}\left(\Phi_{\text {Borel }}\right)
$$

and since the kernel of the Borel regulator is finite (cf. Theorem [5.1), so must be $\operatorname{ker}\left(\mu_{2 n-1}\right)$, and thus the image of $\partial_{2 n}$; for all $n \geq 2$. Moreover, $K_{2 n}(\mathcal{O})$ is finite for all $n \geq 1$, so the image of $\partial_{2 n-1}$ is also finite. It follows that the bottom long exact sequence in Figure 5.3 splits into separate short exact sequences rationally. This is also true for small $n$ : The group $K_{2}(\mathcal{O})$ is finite, so it remains to check

$$
K_{2}\left(\mathrm{LCA}_{\mathcal{O}}\right) \rightarrow K_{1}(\mathcal{O}) \stackrel{\mu_{1}}{\rightarrow} K_{1}(\mathbb{R})^{r} \oplus K_{1}(\mathbb{C})^{s} \rightarrow K_{1}\left(\mathrm{LCA}_{\mathcal{O}}\right) \rightarrow K_{0}(\mathcal{O}),
$$

but $\operatorname{ker}\left(\mu_{1}\right)$ is just the roots of unity, which is a finite group, so $\partial_{2}$ has finite image. Correspondingly,

$$
K_{0}(\mathcal{O}) \cong \mathbb{Z} \oplus \mathrm{Cl}(\mathcal{O}) \stackrel{\mu_{0}}{\longrightarrow} \mathbb{Z}^{r} \oplus \mathbb{Z}^{s},
$$

so again $\mu_{0}$ has finite kernel by the finiteness of the class group. It follows that the entire long exact sequence splits into short exact sequences rationally. 
Theorem 5.5. Let $F$ be a number field and $\mathcal{O}$ its ring of integers. Suppose $n \geq 2$. There is a canoncial Borel regulator map on the torus quotient by the regulator lattice

$$
K_{2 n-1}^{\#}\left(\mathrm{LCA}_{\mathcal{O}}\right) \rightarrow \mathbb{R}^{d_{n}} / \Phi_{\text {Borel }}\left(K_{2 n-1}(\mathcal{O})\right),
$$

where $K_{2 n-1}^{\#}\left(\mathrm{LCA}_{\mathcal{O}}\right)$ is some well-defined finite index subgroup of $K_{2 n-1}\left(\mathrm{LCA}_{\mathcal{O}}\right)$.

Proof. We use the same setup as in the previous proof; with the same notation. Take Figure 5.3. We make $\mu_{2 n-1}$ injective by quotienting out $\operatorname{ker}\left(\mu_{2 n-1}\right)$. By Equation 5.5 the Borel regulator is still well-defined on this quotient. Secondly, we replace $K_{2 n-1}\left(\mathrm{LCA}_{\mathcal{O}}\right)$ by the image of the incoming arrow. This agrees with $\operatorname{ker}\left(\partial_{2 n-1}\right)$, as in

$$
\operatorname{ker}\left(\partial_{2 n-1}\right) \hookrightarrow K_{2 n-1}\left(\mathrm{LCA}_{\mathcal{O}}\right) \rightarrow \text { image in } K_{2 n-2}(\mathcal{O})
$$

and since $K_{2 n-2}(\mathcal{O})$ is a finite group, it follows that this is a finite index subgroup. Now, the universal property of cokernels implies that $\phi$ descends to a morphism

$$
\bar{\phi}: \operatorname{ker}\left(\partial_{2 n-1}\right) \longrightarrow \mathbb{R}^{d_{n}} / \Phi_{\text {Borel }}\left(K_{2 n-1}(\mathcal{O})\right)
$$

and as $\Phi_{\text {Borel }}$ has full rank image, and thus is surjective after tensoring with the reals, $\phi$ and thus $\bar{\phi}$ are surjective.

Example 7. (Classical algebraic number theory) In low degrees, Theorem 4.5 yields the exact sequence

$$
\cdots \rightarrow K_{1}(\mathcal{O}) \rightarrow K_{1}(\mathbb{R})^{r} \oplus K_{1}(\mathbb{C})^{s} \rightarrow K_{1}\left(\mathrm{LCA}_{\mathcal{O}}\right) \rightarrow K_{0}(\mathcal{O}) \rightarrow \mathbb{Z}^{r+s} \rightarrow K_{0}\left(\mathrm{LCA}_{\mathcal{O}}\right) \rightarrow 0
$$

Note that all the "usual suspects" of basic algebraic number theory show up: (1) We have $K_{0}(\mathcal{O}) \cong \mathbb{Z} \oplus \mathrm{Cl}(\mathcal{O})$. Since torsion must map to zero in $\mathbb{Z}^{r+s}$, yet the free rank part maps diagonally to the real and complex vector spaces, the last three terms split off to form

$$
K_{0}\left(\mathrm{LCA}_{\mathcal{O}}\right) \cong \mathbb{Z}^{r+s-1} \text {. }
$$

Thus, $K_{0}\left(\mathrm{LCA}_{\mathcal{O}}\right)$ has the same free rank as the unit group $\mathcal{O}^{\times}$. Moreover, using the BassMilnor-Serre Theorem the middle part of the sequence becomes

$$
K_{2}\left(\mathrm{LCA}_{\mathcal{O}}\right) \stackrel{\nu}{\rightarrow} \mathcal{O}^{\times} \stackrel{\mu}{\rightarrow}\left(\mathbb{R}^{\times}\right)^{r} \oplus\left(\mathbb{C}^{\times}\right)^{s} \rightarrow K_{1}\left(\mathrm{LCA}_{\mathcal{O}}\right) \rightarrow \mathrm{Cl}(\mathcal{O}) \rightarrow 0
$$

and $\mu$ sends a unit $\alpha \in \mathcal{O}^{\times}$to its embedding $\sigma(\alpha)$ in the real or complex numbers. It follows that $\nu=0$. Composing the middle term with a logarithm,

$$
\begin{aligned}
\left(\mathbb{R}^{\times}\right)^{r} \oplus\left(\mathbb{C}^{\times}\right)^{s} & \longrightarrow \mathbb{R}^{r+s} \\
\left(\ldots, x_{i}, \ldots\right) & \longmapsto\left(\ldots, \log \left|\sigma\left(x_{i}\right)\right|, \ldots\right),
\end{aligned}
$$

we thus find both the Dirichlet embedding as well as the class group appear in Equation 5.6.

\section{Comparison with a Tate category}

For background on Tate categories we refer to Pre11, BGW16. Write $\operatorname{Mod}_{f i n}(\mathcal{O})$ for the abelian category of finitely generated Artinian $\mathcal{O}$-modules; or equivalently of $\mathcal{O}$-modules whose underlying set is finite.

Theorem 6.1. Let $K: \mathrm{Cat}_{\infty}^{\mathrm{ex}} \rightarrow \mathrm{A}$ be a localizing invariant. There is a canonical map of fiber sequences in $\mathrm{A}$,

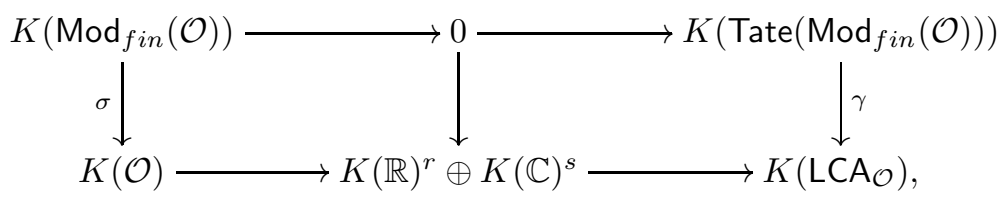


where the bottom row is the sequence of Theorem 4.5, $\sigma$ is induced from the inclusion functor $\operatorname{Mod}_{\text {fin }}(\mathcal{O}) \hookrightarrow \operatorname{Mod}_{f g}(\mathcal{O})$, and $\gamma$ is induced from the evaluation of the ind-pro limit inside $\mathrm{LCA}_{\mathcal{O}}$.

Proof. We return to Diagram 4.1,

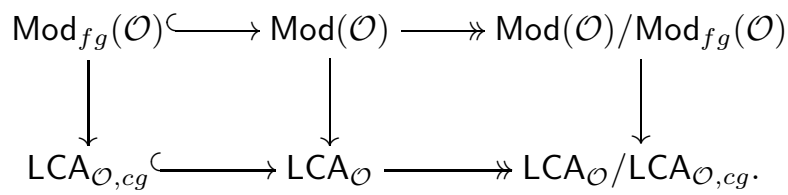

with the exact equivalence of Lemma 4.4 on the right. There is a quite analogous diagram, first constructed by Sho Saito Sai15, end of page 9],

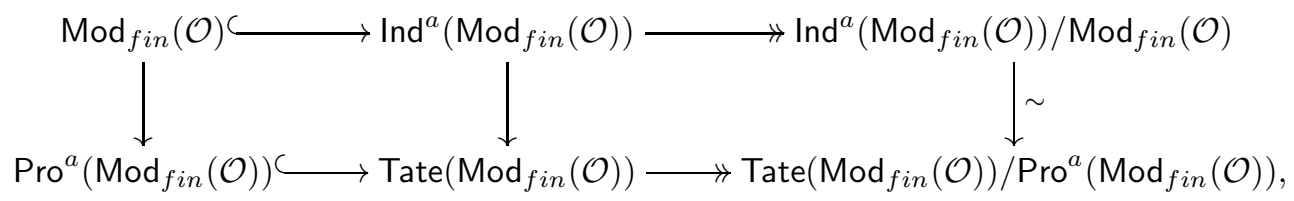

where both rows are exact sequences of exact categories. The right downward arrow is an exact equivalence (Sai15, Lemma 3.3], loc. cit. this is done for countable diagrams, but it works just as well without a cardinality constraint, see [BGW16, Prop. 5.32]). Next, we note that we can find exact functors mapping the second diagram commutatively to the former: On the upper left we just the inclusion $\operatorname{Mod}_{\text {in }}(\mathcal{O}) \subset \operatorname{Mod}_{f g}(\mathcal{O})$. Further, there are exact functors of evaluation

$$
\alpha: \operatorname{Ind}^{a}\left(\operatorname{Mod}_{f i n}(\mathcal{O})\right) \longrightarrow \operatorname{Mod}(\mathcal{O}) \quad \text { and } \quad \beta: \operatorname{Pro}^{a}\left(\operatorname{Mod}_{f i n}(\mathcal{O})\right) \longrightarrow \operatorname{LCA}_{\mathcal{O}, C}
$$

(we justify this: The category $\operatorname{Mod}(\mathcal{O})$ is Grothendieck abelian. Thus, all colimits exist and the evaluation of a colimit is an exact functor. This settles $\alpha$. Note that $\beta$ unravels to be a functor $\beta:$ Ind $^{a}\left(\operatorname{Mod}_{f i n}(\mathcal{O})^{o p}\right)^{o p} \rightarrow \mathrm{LCA}_{\mathcal{O}, C}$, and thus setting it up is equivalent to giving an exact functor $\beta^{o p}: \operatorname{Ind}^{a}\left(\operatorname{Mod}_{f i n}(\mathcal{O})^{o p}\right) \rightarrow \operatorname{LCA}_{\mathcal{O}, D}$. The category $\operatorname{Mod}_{f i n}(\mathcal{O})$ is equivalent to its opposite through Pontryagin duality, and $\mathrm{LCA}_{\mathcal{O}, D} \cong \operatorname{Mod}(\mathcal{O})$, so the same argument as for $\alpha$ settles that $\beta$ exists and is exact. Finally, $\mathrm{LCA}_{\mathcal{O}, C}$ is a full subcategory of $\mathrm{LCA}_{\mathcal{O}, c g}$ ). Moreover, there is a functor of evaluation

$$
\gamma: \operatorname{Tate}^{e l}\left(\operatorname{Mod}_{f i n}(\mathcal{O})\right) \longrightarrow \mathrm{LCA}_{\mathcal{O}}
$$

defined analogously: We use the presentation of $\operatorname{Tate}^{e l}\left(\operatorname{Mod}_{f i n}(\mathcal{O})\right)$ as a full subcategory of Pro $^{a}$ Ind $^{a}\left(\operatorname{Mod}_{f i n}(\mathcal{O})\right)$. Every object can be presented as an admissible Pro diagram $X: I \rightarrow$ Ind $^{a}\left(\operatorname{Mod}_{f i n}(\mathcal{O})\right)$ such that for all arrows in the diagram category $I$, we have $X_{i, j} \hookrightarrow X(i) \rightarrow$ $X(j)$ with $X_{i, j}$ an Artinian $\mathcal{O}$-module. The $X(-)$ are discrete groups. We note that $X(i) \rightarrow$ $X(j)$ is a proper map in the sense of topology, i.e. the preimage of a compact set is compact. This is true since the compact subsets are precisely the finite ones and since the kernel $X_{i, j}$ is finite, the preimage of a finite set is still a finite set, thus compact. It follows that the limit is locally compact, see for example [HM07, Corollary 1.26 (a)] or [Str06, (38.1) Lemma]. As for $\alpha, \beta$ one finds that $\gamma$ is exact. Finally, as $\mathrm{LCA}_{\mathcal{O}}$ is idempotent complete, $\gamma$ canonically extends to the idempotent completions, i.e. Tate $\left(\operatorname{Mod}_{f i n}(\mathcal{O})\right)$ in the case at hand, Büh10, Prop. 6.10]. The functors on the quotient categories on the right in Diagrams 6.1 and 6.2 are induced from these functors and the commutativity of the square on the left. As a result of this, following the 
construction in the proof of Theorem 4.7, we obtain a canonical morphism of fiber sequences

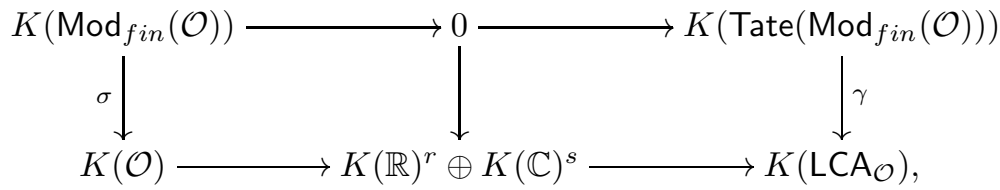

We have used the Eilenberg swindle to get $K\left(\operatorname{Pro}^{a}\left(\operatorname{Mod}_{f i n}(\mathcal{O})\right)\right)=0$. This proves our claim.

Example 8. There is also a variant of Diagram 6.2, replacing $\operatorname{Mod}_{f i n}$ by $\operatorname{Mod}_{f g}$, but in order to map it to LCA groups, we would need functors $\operatorname{Ind}^{a}\left(\operatorname{Mod}_{f g}(\mathcal{O})\right) \rightarrow \operatorname{Mod}(\mathcal{O})$ and $\operatorname{Pro}^{a}\left(\operatorname{Mod}_{f g}(\mathcal{O})\right) \rightarrow$ $\mathrm{LCA}_{\mathcal{O}, c g}$. While the former exists and is even an equivalence of abelian categories, the latter cannot reasonably be set up. For example, for $\mathcal{O}=\mathbb{Z}$ the infinite product $\prod_{\mathbb{Z}} \mathbb{Z}$ fails to be compactly generated. So it is not possible to construct a functor Tate $\left(\operatorname{Mod}_{f g}(\mathcal{O})\right) \rightarrow \operatorname{LCA} \mathcal{O}$ by the method of the above theorem.

Proposition 6.2 (Soulé). For non-connective $K$-theory $\mathbb{K}$, the map $\sigma: \mathbb{K}_{i}\left(\operatorname{Mod}_{\text {fin }}(\mathcal{O})\right) \rightarrow$ $\mathbb{K}_{i}(\mathcal{O})$ of the previous theorem is zero for all integers $i \neq 0$. If $F$ has trivial class group, it is also trivial in degree zero.

Proof. Temporarily write $K$ for connective $K$-theory. Firstly, $\operatorname{Mod}_{f i n}(\mathcal{O})$ is a Noetherian abelian category, so by Schlichting's Connectivity Theorem $\left[\operatorname{Sch06}\right.$, Theorem 7] we have $\mathbb{K}_{i}\left(\operatorname{Mod}_{\text {fin }}(\mathcal{O})\right)=$ 0 for $i<0$; and moreover $\mathbb{K}_{0}\left(\operatorname{Mod}_{\text {fin }}(\mathcal{O})\right)=K_{0}\left(\operatorname{Mod}_{f i n}(\mathcal{O})\right)$, Sch06, Remark 3]; and $\mathbb{K}_{i}\left(\operatorname{Mod}_{\text {fin }}(\mathcal{O})\right)=$ $K_{i}\left(\operatorname{Mod}_{\text {fin }}(\mathcal{O})\right)$ for $i \geq 1$ anyway. Finally, $\mathcal{O}$ is a regular ring, so $\mathbb{K}_{i}(\mathcal{O})=K_{i}(\mathcal{O})$. Thus, it suffices to prove the claim for connective $K$-theory. For this, we follow the presentation in Weibel Wei13, Chapter $\mathrm{V}, \S 6]$ : For every number field $F$ and ring of integers $\mathcal{O}$, we have the exact sequence $\operatorname{Mod}_{f i n}(\mathcal{O}) \hookrightarrow \operatorname{Mod}_{f g}(\mathcal{O}) \rightarrow \operatorname{Mod}_{f g}(F)$ of abelian categories, and the resulting localization sequence reads

$$
\cdots \longrightarrow K_{i+1}(\mathcal{O}) \longrightarrow K_{i+1}(F) \stackrel{\partial_{x}^{F}}{\longrightarrow} K_{i}\left(\operatorname{Mod}_{f i n}(\mathcal{O})\right) \stackrel{\iota_{i}}{\longrightarrow} K_{i}(\mathcal{O}) \longrightarrow \cdots
$$

Dévissage yields

$$
K_{i}\left(\operatorname{Mod}_{f i n}(\mathcal{O})\right) \cong \coprod_{x \in(\operatorname{Spec} \mathcal{O})_{(0)}} K_{i}(\kappa(x)),
$$

the $\kappa(x)$ are finite fields, so by Quillen's computation of the $K$-theory of finite fields we deduce that $\iota_{i}=0$ for $i \geq 2$ even since $K_{i}\left(\mathbb{F}_{q}\right)=0$ for these $i$. On the other hand, Soulé shows that for $i$ odd, $i \geq 1$, the sequence splits into short exact sequences, so that $\partial_{x}^{F}$ is surjective, cf. [Wei13, Ch. V, $\S 6$, Theorem 6.8]. Thus, $\iota_{i}=0$ for $i \geq 1$ odd. For $i=0$, we get $F^{\times} \stackrel{\partial_{x}^{F}}{\longrightarrow} \coprod_{x} \mathbb{Z} \stackrel{\iota_{0}}{\longrightarrow} K_{0}(\mathcal{O}) \stackrel{\alpha}{\longrightarrow} K_{0}(F) \longrightarrow 0$ and $K_{0}(\mathcal{O}) \cong \mathbb{Z} \oplus \mathrm{Cl}(\mathcal{O})$. Thus, as $\alpha$ is the rank map, it follows that $\iota_{0}$ is a surjection on the class group of $F$.

Remark 6.3. Thus, we obtain a lift

$$
\mathbb{K}_{i}\left(\operatorname{Tate}\left(\operatorname{Mod}_{f i n}(\mathbb{Z})\right)\right) \longrightarrow \mathbb{K}_{i}(\mathbb{R}) / \operatorname{im}\left(\mathbb{K}_{i}(\mathbb{Z})\right)
$$

\section{Appendix A. Characterization of injectives And projectives}

Next, we need to recall the structure of the injective and projective objects in LCA . See [Büh10, §11] for a discussion of these concepts in exact categories. For LCA these results are due to Moskowitz [Mos67, and for LCA $\mathcal{O}_{\mathcal{O}}$ due to Kryuchkov Kry97. Unfortunately, Kryuchkov's results appear to be unknown to a wider audience. The paper was published over 20 years ago and seems not to have been cited in any research article since then. We miss out on some interesting results. Below, we give an exposition of how Kryuchkov's more general results can 
be obtained if we take the ones for LCA in Mos67 for granted. We claim no originality for this whatsoever; the strategy follows Mos67 and Kry97.

Lemma A.1. Suppose $I$ is an injective object in $\mathrm{LCA}_{\mathcal{O}}$. Then $I$ is connected and injective as an algebraic $\mathcal{O}$-module.

Proof. Connectedness: Let $y \in I$ be arbitrary. Consider the Minkowski sequence for $\mathcal{O}$ itself, Equation 2.1,

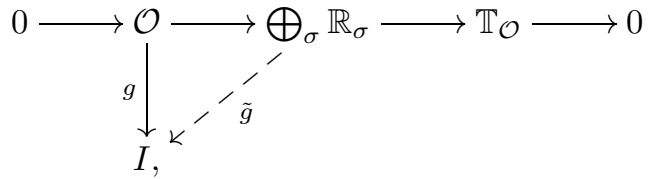

where $g(r):=r y$. As $I$ is injective, $\tilde{g}$ exists. However, $\bigoplus_{\sigma} \mathbb{R}_{\sigma}$ is connected and $\tilde{g}$ continuous, so the image of $\tilde{g}$ lies in the connected component of zero in $I$. However, $y$ lies in this image and $y$ was arbitrary, so this connected component is the entire group. Injectivity: Similarly, consider the sequence $\mathcal{O} \stackrel{\cdot a}{\hookrightarrow} \mathcal{O} \rightarrow \mathcal{O} / a$ with $g: \mathcal{O} \rightarrow I$ as before. As $I$ is injective, we get an analogous lift $\tilde{g}: \mathcal{O} \rightarrow I$ so that $y=g(1)=\tilde{g}(a)=a \tilde{g}(1)$, showing that $y$ is a multiple of $a$. As $y$ was arbitrary, it follows that $I$ is divisible as an $\mathcal{O}$-module and since $\mathcal{O}$ is Dedekind, it is an injective module.

Lemma A.2. Let $I \in \mathrm{LCA}_{\mathcal{O}}$ be an object such that for every diagram given by the solid arrows in

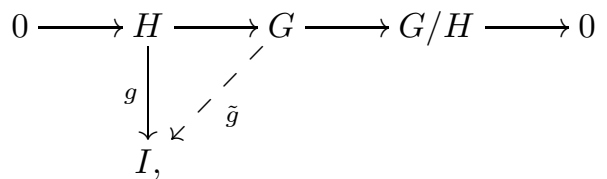

and $G \in \mathrm{LCA}_{\mathcal{O}, c g}$, the dashed lift $\tilde{g}$ exists. Then $I$ is an injective object in $\mathrm{LCA}_{\mathcal{O}}$. That is: Being injective can be detected by being injective for compactly generated modules.

Proof. We sketch the argument for completeness, but the argument is exactly the same as used by Moskowitz and also Kryuchkov: Firstly, we claim that $I$ is injective as an algebraic $\mathcal{O}$ module, without topology. For this copy the argument in the proof of Lemma A.1, noting that it only uses injectivity for exact sequences whose middle term is compactly generated, so by our assumptions the same argument is valid in this case. Secondly, pick a compactly generated clopen $\mathcal{O}$-submodule $L$, which is possible by Lemma 2.14. Then $L \cap H \hookrightarrow L \rightarrow L /(L \cap H)$ is an exact sequence in $\mathrm{LCA}_{\mathcal{O}}$. By assumption the restriction of $f$ to $L \cap H$, call it $f_{1}: L \cap H \rightarrow I$, has a lift $\tilde{f}_{1}: L \rightarrow I$ in LCA . Now consider the exact sequence $L \cap H \hookrightarrow L \oplus H \rightarrow L+H$ in $\mathrm{LCA}_{\mathcal{O}}$ with $x \mapsto(x,-x)$ and the sum map as arrows. Define $f_{2}: L \oplus H \rightarrow I$ by $\tilde{f}_{1}+f$. As this map restricts to the zero map on $L \cap H$, we get a (continuous) factorization

$$
f_{3}: L+H \longrightarrow I \quad \text { with } \quad f_{3}(l+h)=\tilde{f}_{1}(l)+f(h)
$$

for any sum decomposition $l+h$ with $l \in L$ and $h \in H$. We then get a further exact sequence $L+H \hookrightarrow G \rightarrow D$ with $D$ discrete. On the level of algebraic $\mathcal{O}$-modules, as $I$ is injective, there exists an algebraic lift $\tilde{f}_{3}: G \rightarrow H$ extending $f_{3}$. As $L$ was open in $G$, so is $L+H$. Thus, checking continuity of $\tilde{f}_{3}$ amounts to checking it on $L+H$ as $G /(L+H)$ is discrete. However, on $L+H$ it agrees with $f_{3}$, which we know is continuous. Thus, $\tilde{f}_{3}$ is a morphism in LCA $C_{\mathcal{O}}$. By the description of Equation A.1 with $l=0$ it follows that $\left.\tilde{f}_{3}\right|_{H}=f$, so $\tilde{f}_{3}$ is the desired lift. 
Lemma A.3. Let $\nu$ a real or complex place. For every diagram given by the solid arrows in

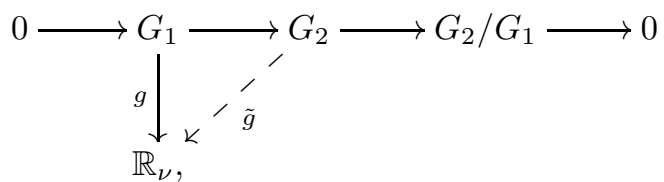

and $G_{2} \in \mathrm{LCA}_{\mathcal{O}, c g}$, a lift $\tilde{g}$ as indicated by the dashed arrow exists.

Proof. (Preparation) Note that closed subgroups of compactly generated LCA groups are also compactly generated, Mos67, Theorem 2.6]. Thus, $G_{1} \in$ LCA $_{\mathcal{O}}$. We apply the structure theorem (Proposition 2.13) to both, writing the compact summand as a subobject, resulting in the solid arrows of the following diagram

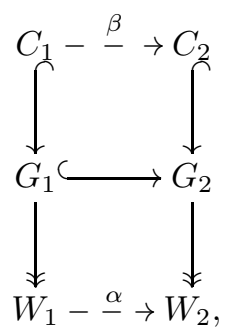

where $W_{i} \simeq \bigoplus_{\sigma \in I_{i}} \mathbb{R}_{\sigma} \oplus \bigoplus_{J \in \mathcal{I}_{i}} J$ for $i=1,2$. Note that $W_{i}$ has no non-trivial compact subgroups (this is classical for $\mathbb{R}$, and each $J$ is a free $\mathbb{Z}$-module). Thus the diagonal map $C_{1} \rightarrow W_{2}$ must be the zero map. Thus, $\beta$ exists by universal property, and thus $\alpha$, giving the dashed arrows. Moreover, $\beta$ is an admissible monic by [Büh10, Cor. 7.7] (applied to the opposite category). Moreover, the kernel of $\alpha$ is $W_{1} \cap C_{2}$ (using that $W_{1}$ is also a subobject), and since $C_{2}$ is compact, this must be the zero object. This does not yet suffice to know that $\alpha$ is an admissible monic: To this end, use that $W_{1}$ is a subobject, so $W_{1} \hookrightarrow G_{1}$ is a closed map, $G_{1} \hookrightarrow G_{2}$ is a closed map, and $G_{2} \rightarrow W_{2}$ is also closed since $C_{2}$ is compact (this really needs compactness; see [Mor77, Prop. 11] for a proof). Hence, the composition $\alpha: W_{1} \rightarrow W_{2}$ is closed. Thus, $\alpha$ is an admissible monic. From here, we proceed by a step of reductions:

(Special Case 1, $C_{1}=C_{2}=0$ and $W_{2}$ is a vector $\mathcal{O}$-module) In this case $G_{i}=W_{i}$. Consider the admissible monic $\alpha: W_{1} \hookrightarrow W_{2}$. We can clearly lift maps along this embedding,

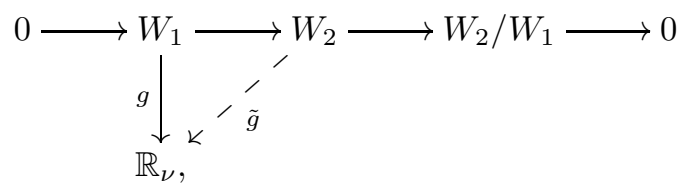

because Proposition 2.12 decomposes it into convenient direct summands: Since lifting along $\mathbb{R}_{\sigma} \stackrel{1}{\rightarrow} \mathbb{R}_{\sigma}$ is trivial, and lifting along $J \hookrightarrow \bigoplus_{\sigma \in I^{\prime}} \mathbb{R}_{\sigma}$ just amounts to extending the map defined on $J$ in an $\mathbb{R}$-linear fashion to the right-hand side, the existence of the lift is clear. So the construction of the lift $\tilde{g}$ just amounts to scalar extension.

(Special Case 2, $C_{1}=C_{2}=0$ ) We have

$$
W_{2}=\bigoplus_{\sigma \in I_{i}} \mathbb{R}_{\sigma} \oplus \bigoplus_{J \in \mathcal{I}_{i}} J
$$

and again by the Minkowski embedding as in Equation 2.1 we may embed $W_{2}$ in a vector $\mathcal{O}_{-}$ module. Thanks to Special Case 1, we already know how to lift a map in the case $W_{2}$ is a vector $\mathcal{O}$-module. Thus, we use this lift and restrict it to $W_{2}$ lying inside of it.

(General Case) Consider $g: G_{1} \rightarrow \mathbb{R}_{\nu}$. Clearly $g\left(C_{1}\right)=0$ since the image of $C_{1}$ must be 
compact, but $\mathbb{R}_{\nu}$ has no non-trivial compact subgroups. Thus, $g$ factors to $W_{1} \rightarrow \mathbb{R}_{\nu}$. By Special Case 2 we already know how to lift maps along $W_{1} \hookrightarrow W_{2}$, so we obtain a lift $\tilde{g}: W_{2} \longrightarrow \mathbb{R}_{\nu}$ and thus are in the situation

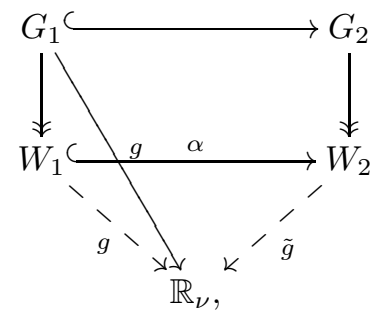

producing a lift to $G_{2}$ by composing the maps along the right edge.

Lemma A.4. Let $J$ be a non-zero ideal of $\mathcal{O}$. For every diagram given by the solid arrows in

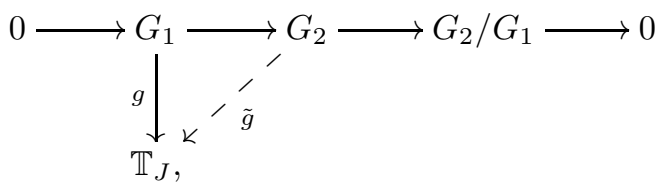

and $G_{2} \in \mathrm{LCA}_{\mathcal{O}, c g}$, a lift $\tilde{g}$ as indicated by the dashed arrow exists.

Proof. Use Pontryagin duality for $\mathcal{O}$-modules, Theorem 2.3, to obtain the diagram

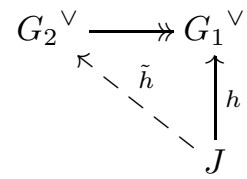

with $h:=g^{\vee}$ since $\mathbb{T}_{J}^{\vee} \simeq J$. Now, forgetting topology, the ideal $J$ is a projective algebraic $\mathcal{O}$-module, so the algebraic $\mathcal{O}$-module lift $\tilde{h}$ exists. Since $J$ is discrete, it is tautologically continuous. Dualizing again, $\tilde{h}^{\vee}$ is the required lift.

Now we are ready to prove the characterization of injectives. For LCA this is due to Moskowitz Mos67] and the variant for number fields is due to Kryuchkov Kry97.

Proof of Theorem [2.16. $(1 \Rightarrow 2)$ Suppose $I$ is an injective object. By Lemma A.1 it is connected. Thus, for the additive group we have an isomorphism

$$
I \simeq \mathbb{R}^{n} \oplus C \quad(\text { in LCA })
$$

for some $n \geq 0$ and $C$ compact connected by the classification of connected LCA groups, Mor77, Theorem 26]. For the dual we get $I^{\vee} \simeq \mathbb{R}^{n} \oplus D$ with $D$ discrete (in LCA), By Lemma 2.11 the direct sum decomposition of Equation A.2 in LCA gets promoted to an isomorphism $I^{\vee} \simeq \bigoplus_{\sigma \in I} \mathbb{R}_{\sigma} \oplus D$ in $\mathrm{LCA}_{\mathcal{O}}$. Hence, dualizing again, $I \cong \bigoplus_{\sigma \in I} \mathbb{R}_{\sigma} \oplus C$ in LCA $\mathrm{L}_{\mathcal{O}}$. Now as $I$

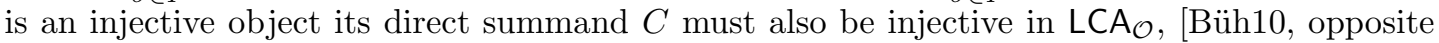
of Cor. 11.6]. Thus, $C^{\vee}$ is a projective object, but as $C$ is compact, it is discrete. Thus, $C^{\vee}$ is a projective as an algebraic $\mathcal{O}$-module. Since $\mathcal{O}$ is a Dedekind domain, the classification of projective modules yields an isomorphism $C^{\vee} \simeq \bigoplus_{J \in \mathcal{I}} J$ for a possibly infinite list $\mathcal{I}$ of ideals (Details: Dedekind domains are hereditary rings, so this claim follows from Lam99, §2E, (2.24) Theorem and (2.25)]). As the topology on $C^{\vee}$ is discrete, this tautologically also holds in LCA $\mathcal{O}$. Thus, by dualizing, $C \simeq \prod_{J \in \mathcal{I}} \mathbb{T}_{J}$.

$(2 \Rightarrow 3)$ The underlying additive group is $\mathbb{R}^{n} \oplus \mathbb{T}^{\omega}$ for some $n \geq 0$ and $\omega$ some cardinal. By Moskowitz [Mos67, Theorem 3.2] this is an injective object in LCA. Moreover, each $\mathbb{R}_{\sigma}$ is also an $\mathrm{F}$-module, so they are divisible $\mathcal{O}$-modules, thus injective as $\mathcal{O}$ is Dedekind. Similarly, 
each $\mathbb{T}_{J}$ is divisible (use the surjection from the $F$-modules $\mathbb{R}_{\sigma}$ in Equation 2.1), and arbitrary products of injective objects are injective, Büh10, opposite of Cor. 11.7].

$(3 \Rightarrow 2)$ The underlying additive group is injective in LCA and thus using [Mos67, Theorem 3.2] isomorphic to $\mathbb{R}^{n} \oplus \mathbb{T}^{\omega}$ for some $n \geq 0$ and $\omega$ some cardinal in the category LCA. Dualize to get $G^{\vee} \simeq \mathbb{R}^{n} \oplus \bigoplus_{\omega} \mathbb{Z}$. By Lemma 2.11 this implies that $G^{\vee} \simeq \bigoplus_{\sigma \in I} \mathbb{R}_{\sigma} \oplus D$ in LCA $\mathcal{O}_{\mathcal{O}}$ with $D$ discrete. By assumption the underlying algebraic $\mathcal{O}$-module of $G$ is injective, thus the one of $G^{\vee}$ must be projective. Thus, so must be its direct summands and hence $D$ is a projective $\mathcal{O}$-module. Hence, it is a possible infinite direct sum of ideals of $\mathcal{O}$. Dualizing again, we obtain

$$
G \simeq \bigoplus_{\sigma \in I} \mathbb{R}_{\sigma} \oplus \prod_{J \in \mathcal{I}} \mathbb{T}_{J}
$$

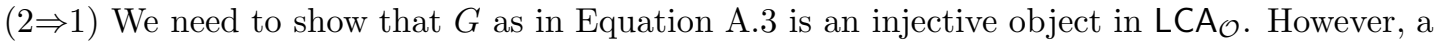
product of injective objects is always again injective, so it suffices to show that the factors $\mathbb{R}_{\sigma}$ (for any real or complex place $\sigma$ ) resp. $\mathbb{T}_{J}$ for any ideal $J$ are injective in LCA $\mathcal{O}_{\mathcal{O}}$. Secondly, by Lemma A.2 it suffices to check this for sequences $G_{1} \hookrightarrow G_{2} \rightarrow G_{2} / G_{1}$, where $G_{2}$ is in LCA $\mathrm{L}_{\mathcal{O}, c g}$. However, these two cases are handled by Lemma A.3 and Lemma A.4 respectively. Thus, $G$ is indeed an injective object.

Acknowledgement. I heartily thank Dustin Clausen for several discussions; his work started the entire project and was a great source of inspiration. Moreover, I thank Dmitri Shakhmatov, Markus Spitzweck, Nikolay Kryuchkov, Martin Levin, and Matthias Wendt for very helpful correspondence and encouragement. Moreover, we thank the FRIAS for providing excellent working conditions.

\section{REFERENCES}

[BG02] J. Burgos Gil, The regulators of Beilinson and Borel, CRM Monograph Series, vol. 15, American Mathematical Society, Providence, RI, 2002. MR 18696555

[BGT13] A. Blumberg, D. Gepner, and G. Tabuada, A universal characterization of higher algebraic $K$-theory, Geom. Topol. 17 (2013), no. 2, 733-838. MR 3070515 14

[BGW16] O. Braunling, M. Groechenig, and J. Wolfson, Tate objects in exact categories, Mosc. Math. J. 16 (2016), no. 3, 433-504, With an appendix by Jan Šťovíček and Jan Trlifaj. MR 3510209 3 3 6 6

[Büh10] T. Bühler, Exact categories, Expo. Math. 28 (2010), no. 1, 1-69. MR 2606234 (2011e:18020) 1 20 $3,3,6 \mathrm{AA}$

[Cla17] D. Clausen, A K-theoretic approach to Artin maps, arXiv:1703.07842 [math.KT] (2017). 11 1 4 4

[FS02] H. Fujita and D. Shakhmatov, Topological groups with dense compactly generated subgroups, Appl. Gen. Topol. 3 (2002), no. 1, 85-89. MR 1931255 [

[HM07] K. H. Hofmann and S. A. Morris, The Lie theory of connected pro-Lie groups, EMS Tracts in Mathematics, vol. 2, European Mathematical Society (EMS), Zürich, 2007, A structure theory for pro-Lie algebras, pro-Lie groups, and connected locally compact groups. MR 2337107 6

[HS07] N. Hoffmann and M. Spitzweck, Homological algebra with locally compact abelian groups, Adv. Math. 212 (2007), no. 2, 504-524. MR 2329311 (2009d:22006) 122

[Kel96] B. Keller, Derived categories and their uses, Handbook of algebra, Vol. 1, North-Holland, Amsterdam, 1996, pp. 671-701. MR 1421815 (98h:18013) 4

[Kry97] N. I. Kryuchkov, Injective and projective objects in the category of locally compact modules over the ring of integers of a global field, Mat. Zametki 62 (1997), no. 1, 118-123. MR 1620000 2.16 2.12.17. $2.20 \mathrm{~A} \mathrm{~A}$

[Lam99] T. Y. Lam, Lectures on modules and rings, Graduate Texts in Mathematics, vol. 189, Springer-Verlag, New York, 1999. MR 1653294 (99i:16001) A

[Lev73] M. Levin, Locally compact modules, J. Algebra 24 (1973), 25-55. MR 0310125 22.1 2.1,2.11 2.13 2.12 .15

[Mor77] S. Morris, Pontryagin duality and the structure of locally compact abelian groups, Cambridge University Press, Cambridge-New York-Melbourne, 1977, London Mathematical Society Lecture Note Series, No. 29. MR 0442141 2.1 2.1 2.1 3 A

[Mos67] M. Moskowitz, Homological algebra in locally compact abelian groups, Trans. Amer. Math. Soc. 127 (1967), 361-404. MR 0215016 2.1 2.12.12.1 3, A, A A 
[Nik98] N. K. Nikolski (ed.), Commutative harmonic analysis. II, Encyclopaedia of Mathematical Sciences, vol. 25, Springer-Verlag, Berlin, 1998, Group methods in commutative harmonic analysis, A translation of it Current problems in mathematics. Fundamental directions. Vol. 25 (Russian) Akad. Nauk SSSR, Vsesoyuz. Inst. Nauchn. i Tekhn. Inform., Moscow, 1988 [ MR0982752 (89h:43001)], Translated by D. Dynin and S. Dynin, Translation edited by V. P. Havin [Viktor Petrovich Khavin] and N. K. Nikolski [Nikolai K. Nikol/skiı̌]. MR 16224893

[Pre11] L. Previdi, Locally compact objects in exact categories, Internat. J. Math. 22 (2011), no. 12, 17871821. MR 28725336

[Rap88] M. Rapoport, Comparison of the regulators of Bellinson and of Borel, Beillinson's conjectures on special values of $L$-functions, Perspect. Math., vol. 4, Academic Press, Boston, MA, 1988, pp. 169192. MR 9449945

[Sai15] S. Saito, On Previdi's delooping conjecture for K-theory, Algebra Number Theory 9 (2015), no. 1, 1-11. MR 3317759 6 6

[Sch04] M. Schlichting, Delooping the K-theory of exact categories, Topology 43 (2004), no. 5, 1089-1103. MR 2079996 (2005k:18023) 3 4

[Sch06] _ Negative K-theory of derived categories, Math. Z. 253 (2006), no. 1, 97-134. MR 22066396

[Sch10] , Hermitian K-theory of exact categories, J. K-Theory 5 (2010), no. 1, 105-165. MR 2600285 2.4

[Sto69] K. O. Stoehr, Dualitäten in der Kategorie der lokal kompakten Moduln über einem Dedekindschen Ring, J. Reine Angew. Math. 239/240 (1969), 239-255. MR 02622232

[Str06] M Stroppel, Locally compact groups, EMS Textbooks in Mathematics, European Mathematical Society (EMS), Zürich, 2006. MR 2226087 6

[Wei13] C. Weibel, The K-book, Graduate Studies in Mathematics, vol. 145, American Mathematical Society, Providence, RI, 2013, An introduction to algebraic $K$-theory. MR 3076731 [ 6

Freiburg Institute for Advanced Studies (FRIAS), University of Freiburg, D-79104 Freiburg im Breisgau, Germany 